\title{
On a three step crisis integro-differential equation
}

\author{
Dumitru Baleanu ${ }^{1,2}$, Khadijeh Ghafarnezhad ${ }^{3}$ and Shahram Rezapour ${ }^{3 *}$
}

"Correspondence:

rezapourshahram@yahoo.ca

${ }^{3}$ Department of Mathematics, Azarbaijan Shahid Madani

University, Tabriz, Iran

Full list of author information is

available at the end of the article (c) The Author(s) 2019. This article is distributed under the terms of the Creative Commons Attribution 4.0 International License (http://creativecommons.org/licenses/by/4.0/), which permits unrestricted use, distribution, and reproduction in any medium, provided you give appropriate credit to the original author(s) and the source, provide a link to the Creative Commons license, and indicate if changes were made.

\begin{abstract}
One of the interesting fractional integro-differential equations is the three step crisis equation which has been reviewed recently. In this paper, we investigate the existence of solutions for a three step crisis fractional integro-differential equation under some boundary conditions.
\end{abstract}

MSC: Primary 34A08; secondary 34A60

Keywords: Caputo derivation; Pointwise defined equation; Three steps crisis equation; Singularity

\section{Preliminaries}

It is well known that we can make better exact models for most natural phenomena by using fractional differential equations. Most researchers are working on fractional integrodifferential equations (see, for example, $[1,2,5-8,10-21]$ ).

In 2010, Agarwal et al. reviewed the existence of solutions $D^{\alpha} u(t)+f(t, u(t))=0$ with boundary conditions $u^{\prime}(0)=\cdots=u^{(n-1)}=0$ and $u(1)=\int_{0}^{1} u(s) d \mu(s)$, where $n \geq 2, \alpha \in$ $(n-1, n), \mu(s)$ is a functional of bounded variation, $f$ may have singularity at $t=0$ and $\int_{0}^{1} d \mu(s)<1$ [3]. In 2012, Agarwal et al. studied positive solutions for the integral value problem $D^{\alpha} u_{i}(t)+f_{i}\left(t, u_{1}(t), u_{2}(t)\right)=0$ with boundary conditions $u_{i}(0)=u_{i}^{\prime}(0)=0$ and $u_{i}(1)=\int_{0}^{1} u_{i}(t) d \eta(t)$ for $i=1,2$, where $t \in(0,1), \alpha \in(2,3], D^{\alpha}$ is the Riemann-Liouville fractional derivative of order $\alpha, f_{i}$ is a real valued continuous map on $[0,1] \times \mathbb{R}^{+} \times \mathbb{R}^{+}$ and $\int_{0}^{1} u_{i}(t) d \eta(t)$ denotes the Riemann-Stieltjes integral [4]. In 2013, the singular fractional problem $D^{\alpha} u+f\left(t, u, D^{\gamma} u, D^{\mu} u\right)+g\left(t, u, D^{\gamma} u, D^{\mu} u\right)=0$ with boundary conditions $u(0)=u^{\prime}(0)=u^{\prime \prime}(0)=u^{\prime \prime \prime}(0)=0$ was reviewed, where $3<\alpha<4,0<\gamma<1,1<\mu<2, D^{\alpha}$ is the Caputo fractional derivative and $f$ is a Caratheodory function on $[0,1] \times(0, \infty)^{3}[9]$.

Recently, the authors introduced a new model for investigating the fractional differential equations called three step crisis integro-differential equations [11]. By using the idea, we investigate the existence of solutions for the three step crisis integro-differential equation

$$
D^{\alpha} x(t)+f\left(t, x(t), x^{\prime}(t), D^{\beta} x(t), \int_{0}^{t} h(\xi) x(\xi) d \xi\right)=0
$$

with boundary conditions $x(0)=x^{\prime}\left(T_{0}\right), x(1)=x^{\prime}\left(T_{1}\right)$ and $x^{\prime \prime}(0)=x^{(n)}(0)=0$, where $\alpha>1$ with $n=[\alpha]-1, T_{0}, T_{1}, \beta, \lambda, \mu \in(0,1), h \in L^{1}[0,1], D^{\alpha}$ is the Caputo fractional derivative of order $\alpha, f\left(t, x_{1}(t), \ldots, x_{5}(t)\right)=f_{1}\left(t, x_{1}(t), \ldots, x_{4}(t)\right)$ on $[0, \lambda), f\left(t, x_{1}(t), \ldots, x_{5}(t)\right)=$ 
$f_{2}\left(t, x_{1}(t), \ldots, x_{4}(t)\right)$ on $[\lambda, \mu]$ and $f\left(t, x_{1}(t), \ldots, x_{5}(t)\right)=f\left(t, x_{1}(t), \ldots, x_{4}(t)\right)$ on $(\mu, 1]$ in which $f_{1}(t, \cdot, \cdot, \cdot, \cdot)$ and $f_{3}(t, \cdot, \cdot, \cdot, \cdot)$ are continuous on $[0, \lambda)$ and $(\mu, 1]$, respectively, and $f_{2}(t, \cdot, \cdot, \cdot, \cdot)$ is singular at some points $t \in[\lambda, \mu]$. In this case, we use the symbol $f=\left[f_{1}, f_{2}, f_{3}, \lambda, \mu\right][11]$.

As is well known, the Caputo fractional derivative of order $\alpha>0$ of a function $f$ : $(0, \infty) \rightarrow \mathbb{R}$ is defined by ${ }^{c} D^{\alpha} f(t)=\frac{1}{\Gamma(n-\alpha)} \int_{0}^{t} \frac{f^{n}(s)}{(t-s)^{\alpha+1-n}} d s$, where $n=[\alpha]+1$ (see, for example, [13]). Let $\Psi$ be the family of nondecreasing functions $\psi:[0, \infty) \rightarrow[0, \infty)$ such that $\sum_{n=1}^{\infty} \psi^{n}(t)<\infty$ for all $t>0$ [22]. One can check that $\psi(t)<t$ for all $t>0$ [22]. Let $T: X \rightarrow X$ and $\alpha: X \times X \rightarrow[0, \infty)$ be two maps. Then $T$ is called an $\alpha$-admissible map whenever $\alpha(x, y) \geq 1$ implies $\alpha(T x, T y) \geq 1$ [22]. Let $(X, d)$ be a complete metric space, $\psi \in \Psi$ and $\alpha: X \times X \rightarrow[0, \infty)$ a map. A self-map $T: X \rightarrow X$ is called an $\alpha$ - $\psi$-contraction whenever $\alpha(x, y) d(T x, T y) \leq \psi(d(x, y))$ for all $x, y \in X$ [22]. We need the following results.

Lemma 1 ([23]) Let $0<n-1 \leq \alpha<n$. Then $I^{\alpha} D^{\alpha} x(t)=x(t)+\sum_{i=0}^{n-1} c_{i} t^{i}$ for some constants $c_{0}, \ldots, c_{n-1}$.

Lemma 2 ([24]) If $E$ is a closed, bounded and convex subset of a Banach space $X$ and $T: E \rightarrow E$ is completely continuous, then $T$ has a fixed point in $E$.

Lemma 3 ([22]) Let $(X, d)$ be a complete metric space, $\psi \in \Psi, \alpha: X \times X \rightarrow[0, \infty)$ a map and $T: X \rightarrow X$ an $\alpha$-admissible $\alpha-\psi$-contraction. If $T$ is continuous and there exists $x_{0} \in X$ such that $\alpha\left(x_{0}, T x_{0}\right) \geq 1$, then $T$ has a fixed point.

\section{Main results}

Now, we are ready to state and prove our main results.

Lemma 4 Let $\alpha>1, n=[\alpha]+1, T_{0}, T_{1} \in(0,1)$ and $f \in L^{1}[0,1]$. Then $x(t)=\int_{0}^{1} G(t, s) f(s) d s$ is the solution of the pointwise defined equation $D^{\alpha} x(t)+f(t)=0$ with boundary conditions $x(0)=x^{\prime}\left(T_{0}\right), x(1)=x^{\prime}\left(T_{1}\right)$ and $x^{\prime \prime}(0)=\cdots=x^{(n-1)}(0)=0$, where $G(t, s)=\frac{-(t-s)^{\alpha-1}}{\Gamma(\alpha)}+$ $\frac{(1+t)(1-s)^{\alpha-1}}{\Gamma(\alpha)}-\frac{(1+t)\left(T_{1}-s\right)^{\alpha-2}}{\Gamma(\alpha-1)}-\frac{t\left(T_{0}-s\right)^{\alpha-2}}{\Gamma(\alpha-1)}$ whenever $0 \leq s \leq t, s \leq T_{0}, G(t, s)=\frac{-(t-s)^{\alpha-1}}{\Gamma(\alpha)}+$ $\frac{(1+t)(1-s)^{\alpha-1}}{\Gamma(\alpha)}-\frac{(1+t)\left(T_{1}-s\right)^{\alpha-2}}{\Gamma(\alpha-1)}$ whenever $0 \leq T_{0} \leq s \leq t, s \leq T_{1}, G(t, s)=\frac{(1+t)(1-s)^{\alpha-1}}{\Gamma(\alpha)}-\frac{(1+t)\left(T_{1}-s\right)^{\alpha-2}}{\Gamma(\alpha-1)}$ whenever $0 \leq t \leq s \leq T_{1}, s \geq T_{0}, G(t, s)=\frac{(1+t)(1-s)^{\alpha-1}}{\Gamma(\alpha)}-\frac{(1+t)\left(T_{1}-s\right)^{\alpha-2}}{\Gamma(\alpha-1)}-\frac{t\left(T_{0}-s\right)^{\alpha-2}}{\Gamma(\alpha-1)}$ whenever $0 \leq t \leq s \leq T_{0} \leq T_{1}, G(t, s)=\frac{-(t-s)^{\alpha-1}}{\Gamma(\alpha)}+\frac{(1+t)(1-s)^{\alpha-1}}{\Gamma(\alpha)}$ whenever $0 \leq T_{0} \leq T_{1} \leq s \leq t$ and $G(t, s)=\frac{(1+t)(1-s)^{\alpha-1}}{\Gamma(\alpha)}$ whenever $0 \leq t \leq s, s \geq T_{1}$.

Proof Suppose that the equation $D^{\alpha} x(t)+f(t)=0$ holds for all $t \in E \subset[0,1]$, where $m\left(E^{c}\right)=$ 0 and $m$ is the Lebesgue measure on $\mathbb{R}$. Let $f_{0}$ be a function such that $f_{0}=f$ on $E$. It is easy to check that $I^{\alpha}(f(t))=I^{\alpha}\left(f_{0}(t)\right)$ for all $t \in[0,1]$. This implies that $I^{\alpha}\left(D^{\alpha} x(t)\right)=I^{\alpha}\left(-f_{0}(t)\right)$ and by using Lemma 1 we get $x(t)=-\frac{1}{\Gamma(\alpha)} \int_{0}^{t}(t-s)^{\alpha-1} f(s) d s+c_{0}+c_{1} t$ for some constants $c_{0}$ and $c_{1}$. By using the boundary conditions, we obtain $x(0)=c_{0}$ and

$$
x^{\prime}\left(T_{0}\right)=-\frac{1}{\Gamma(\alpha-1)} \int_{0}^{T_{0}}\left(T_{0}-s\right)^{\alpha-1} f(s) d s+c_{1} .
$$

Thus, $c_{1}-c_{0}=-\frac{1}{\Gamma(\alpha-1)} \int_{0}^{T_{0}}\left(T_{0}-s\right)^{\alpha-1} f(s) d s$. Since $x(1)=x^{\prime}\left(T_{1}\right)$, we get

$$
c_{0}=\frac{1}{\Gamma(\alpha-1)} \int_{0}^{1}(1-s)^{\alpha-1} f(s) d s-\frac{1}{\Gamma(\alpha-1)} \int_{0}^{T_{1}}\left(T_{1}-s\right)^{\alpha-1} f(s) d s
$$


and so

$$
\begin{aligned}
c_{1}= & \frac{1}{\Gamma(\alpha)} \int_{0}^{1}(1-s)^{\alpha-1} f(s) d s \\
& -\frac{1}{\Gamma(\alpha-1)} \int_{0}^{T_{1}}\left(T_{1}-s\right)^{\alpha-2} f(s) d s-\frac{1}{\Gamma(\alpha-1)} \int_{0}^{T_{0}}\left(T_{0}-s\right)^{\alpha-2} f(s) d s .
\end{aligned}
$$

Hence,

$$
\begin{aligned}
x(t)= & -\frac{1}{\Gamma(\alpha)} \int_{0}^{t}(t-s)^{\alpha-1} f(s) d s-\frac{1}{\Gamma(\alpha)} \int_{0}^{1}(1-s)^{\alpha-1} f(s) d s \\
& -\frac{1}{\Gamma(\alpha-1)} \int_{0}^{T_{1}}\left(T_{1}-s\right)^{\alpha-2} f(s) d s+\frac{t}{\Gamma(\alpha)} \int_{0}^{1}(1-s)^{\alpha-1} f(s) d s \\
& -\frac{t}{\Gamma(\alpha-1)} \int_{0}^{T_{1}}\left(T_{1}-s\right)^{\alpha-2} f(s) d s-\frac{t}{\Gamma(\alpha-1)} \int_{0}^{T_{0}}\left(T_{0}-s\right)^{\alpha-2} f(s) d s \\
= & -\frac{1}{\Gamma(\alpha)} \int_{0}^{t}(t-s)^{\alpha-1} f(s) d s-\frac{1+t}{\Gamma(\alpha)} \int_{0}^{1}(1-s)^{\alpha-1} f(s) d s \\
& -\frac{1+t}{\Gamma(\alpha-1)} \int_{0}^{T_{1}}\left(T_{1}-s\right)^{\alpha-2} f(s) d s-\frac{t}{\Gamma(\alpha-1)} \int_{0}^{T_{0}}\left(T_{0}-s\right)^{\alpha-2} f(s) d s .
\end{aligned}
$$

Now it is easy to check that $x(t)=\int_{0}^{1} G(t, s) f(s) d s$, where $G$ is the given Green function.

By using some usual calculations, we find that $|G(t, s)| \leq \frac{2+\alpha+T_{0}}{\Gamma(\alpha)}(1-s)^{\alpha-2}$ for all $t, s \in[0,1]$ and $\left|\frac{\partial G}{\partial t}(t, s)\right| \leq \frac{3 \alpha}{\Gamma(\alpha)}(1-s)^{\alpha-2}$ for all $t, s \in[0,1]$. Also, it is easy to see that $D^{\mu} x \in C[0,1]$ and $\left|D^{\mu} x\right| \leq \frac{\left\|x^{\prime}\right\|}{\Gamma(2-\mu)}$ whenever $x \in C^{1}[0,1]$. Here, $0 \leq \mu \leq 1$. Now, consider the Banach space $X=C^{1}[0,1]$ with the norm $\|x\|_{*}=\max \left\{\|x\|,\left\|x^{\prime}\right\|\right\},\|\cdot\|$ is the sup norm on $C[0,1]$. Assume that $f=\left[f_{1}, f_{2}, f_{3}, \lambda, \mu\right]$. Define $T: X \rightarrow X$ by

$$
\begin{aligned}
T_{x}(t)= & \int_{0}^{1} G(t, s) f\left(s, x(s), x^{\prime}(s), D^{\beta} x(s), \int_{0}^{s} h(\xi) x(\xi) d \xi, \phi(x(s))\right) d s \\
= & -\frac{1}{\Gamma(\alpha)} \int_{0}^{t}(t-s)^{\alpha-1} f\left(s, x(s), x^{\prime}(s), D^{\beta} x(s), \int_{0}^{s} h(\xi) x(\xi) d \xi, \phi(x(s)) d s\right. \\
& -\frac{1+t}{\Gamma(\alpha)} \int_{0}^{1}(1-s)^{\alpha-1} f\left(s, x(s), x^{\prime}(s), D^{\beta} x(s), \int_{0}^{s} h(\xi) x(\xi) d \xi, \phi(x(s)) d s\right. \\
& -\frac{1+t}{\Gamma(\alpha-1)} \int_{0}^{T_{1}}\left(T_{1}-s\right)^{\alpha-2} f\left(s, x(s), x^{\prime}(s), D^{\beta} x(s), \int_{0}^{s} h(\xi) x(\xi) d \xi, \phi(x(s)) d s\right. \\
& -\frac{t}{\Gamma(\alpha-1)} \int_{0}^{T_{0}}\left(T_{0}-s\right)^{\alpha-2} f\left(s, x(s), x^{\prime}(s), D^{\beta} x(s), \int_{0}^{s} h(\xi) x(\xi) d \xi, \phi(x(s)) d s\right.
\end{aligned}
$$

for all $x \in X$ and $t \in[0,1]$. Note that the singular pointwise defined problem (1) has a solution if and only if $T$ has a fixed point in $X$. We are going to investigate the singular pointwise defined problem (1) under two different conditions. Here. we present first one. In our second result we denote the map $T$ by $F$.

Theorem 5 Let $f=\left[f_{1}, f_{2}, f_{3}, \lambda, \mu\right], f_{1}(t, 0,0,0,0,0)=0, f_{2}(s, 0,0,0,0,0)=0$ and $f_{3}(u, 0,0,0$, $0,0)=0$ for all $t \in[0, \lambda], s \in[0, \lambda]$ and $u \in[\mu, 1]$. Assume that there are nondecreasing maps 
$\Lambda, \Lambda^{\prime}: X \rightarrow[0, \infty)$ and mappings $a_{1}, a_{2}, a_{3}, a_{4}:(\lambda, \mu) \rightarrow[0, \infty)$ such that $\lim _{z \rightarrow 0^{+}} \frac{\Lambda(z)}{z}=q<$ $\infty, \lim _{z \rightarrow 0^{+}} \frac{\Lambda^{\prime}(z)}{z}=q<\infty$ and $\hat{a_{1}}, \hat{a_{2}}, \hat{a_{3}}, \hat{a}_{4} \in L^{1}[\lambda, \mu]$, where $\hat{a}_{i}=(1-s)^{\alpha-2}$ for $i=1,2,3,4$. Suppose that $\left|f_{1}\left(t, x_{1}, \ldots, x_{5}\right)-f_{1}\left(t, y_{1}, \ldots, y_{5}\right)\right| \leq \sum_{i=1}^{4} \Lambda\left(\left|x_{i}-y_{i}\right|\right)$,

$$
\left|f_{2}\left(t, x_{1}, \ldots, x_{5}\right)-f_{2}\left(t, y_{1}, \ldots, y_{5}\right)\right| \leq \sum_{i=1}^{4} a_{i}(t)\left|x_{i}-y_{i}\right|
$$

and $\left|f_{3}\left(t, x_{1}, \ldots, x_{5}\right)-f_{3}\left(t, y_{1}, \ldots, y_{5}\right)\right| \leq \sum_{i=1}^{4} \Lambda^{\prime}\left(\left|x_{i}-y_{i}\right|\right)$ for almost all $t \in[0,1]$ and every $x_{1}, x_{2}, \ldots, x_{5}, y_{1}, y_{2}, \ldots, y_{5} \in X$. If

$$
\frac{4 q}{\alpha-1}\left(1-(1-\lambda)^{\alpha-1}\right)+\sum_{i=1}^{4}\left\|\hat{a}_{i}\right\|_{[\lambda, \mu]}+\frac{4 q^{\prime}}{\alpha-1}(1-\mu)^{\alpha-1}<\frac{\Gamma(\alpha)}{l \theta_{0}},
$$

then the pointwise defined equation (1) with boundary conditions has a solution, where $\|h\|_{1}=m_{0}, l=\max \left\{1, \frac{1}{\Gamma(2-\beta)}, m_{0}\right\}$ and $\theta_{0}=\max \left\{3 \alpha, 2+\alpha+T_{0}\right\}$.

Proof Let $x_{1}, x_{2} \in X$ and $t \in[0,1]$. Then we have

$$
\begin{aligned}
\left|T_{x_{1}}(t)-T_{x_{2}}(t)\right| \leq & \int_{0}^{1}|G(t, s)| f\left(s, x_{1}(s), x_{1}^{\prime}(s), D^{\beta} x_{1}(s), \int_{0}^{s} h(\xi) x_{1}(\xi) d \xi\right) \\
& -f\left(s, x_{2}(s), x_{2}^{\prime}(s), D^{\beta} x_{2}(s), \int_{0}^{s} h(\xi) x_{2}(\xi) d \xi\right) \mid d s \\
\leq & \int_{0}^{\lambda}|G(t, s)| f_{1}\left(s, x_{1}(s), x_{1}^{\prime}(s), D^{\beta} x_{1}(s), \int_{0}^{s} h(\xi) x_{1}(\xi) d \xi\right) \\
& -f_{1}\left(s, x_{2}(s), x_{2}^{\prime}(s), D^{\beta} x_{2}(s), \int_{0}^{s} h(\xi) x_{2}(\xi) d \xi\right) \mid d s \\
& +\int_{\lambda}^{\mu}|G(t, s)| f_{2}\left(s, x_{1}(s), x_{1}^{\prime}(s), D^{\beta} x_{1}(s), \int_{0}^{s} h(\xi) x_{1}(\xi) d \xi\right) \\
& -f_{2}\left(s, x_{2}(s), x_{2}^{\prime}(s), D^{\beta} x_{2}(s), \int_{0}^{s} h(\xi) x_{2}(\xi) d \xi\right) \mid d s \\
& +\int_{\mu}^{1}|G(t, s)| \mid f_{3}\left(s, x_{1}(s), x_{1}^{\prime}(s), D^{\beta} x_{1}(s), \int_{0}^{s} h(\xi) x_{1}(\xi) d \xi\right) \\
& \times f_{3}\left(s, x_{2}(s), x_{2}^{\prime}(s), D^{\beta} x_{2}(s), \int_{0}^{s} h(\xi) x_{2}(\xi) d \xi\right) \mid d s \\
\leq & \int_{0}^{\lambda}|G(t, s)|\left[\Lambda\left(\left|x_{1}(s)-x_{2}(s)\right|\right)+\Lambda\left(\left|x_{1}^{\prime}(s)-x_{2}^{\prime}(s)\right|\right)\right. \\
& \left.+\Lambda\left(\left|D^{\beta}\left(x_{1}-x_{2}\right)(s)\right|\right)+\Lambda\left(\left|\int_{0}^{s} h(\xi)\left(x_{1}(\xi)-x_{2}(\xi)\right) d \xi\right|\right)\right] d s \\
& +\int_{\mu}^{\mu}|G(t, s)|\left[\Lambda^{\prime}\left(\left|x_{1}(s)-x_{2}(s)\right|\right)+\Lambda^{\prime}\left(\left|x_{1}^{\prime}(s)-x_{2}^{\prime}(s)\right|\right)\right. \\
& \left.+a_{3}(s)\left|D^{\beta}\left(x_{1}-x_{2}\right)(s)\right|+a_{4}(s)\left|\int_{0}^{s} h(\xi)\left(x_{1}(\xi)-x_{2}(\xi)\right) d \xi\right|\right] d s \\
& \int_{1}(s)-x_{2}(s)\left|+a_{2}(s)\right| x_{1}^{\prime}(s)-x_{2}^{\prime}(s) \mid \\
& \\
&
\end{aligned}
$$




$$
\begin{aligned}
& \left.+\Lambda^{\prime}\left(\left|D^{\beta}\left(x_{1}-x_{2}\right)(s)\right|\right)+\Lambda^{\prime}\left(\left|\int_{0}^{s} h(\xi)\left(x_{1}(\xi)-x_{2}(\xi)\right) d \xi\right|\right)\right] d s \\
& \leq \int_{0}^{\lambda}|G(t, s)|\left[\Lambda\left(\left\|x_{1}-x_{2}\right\|\right)+\Lambda\left(\left\|x_{1}^{\prime}-x_{2}^{\prime}\right\|\right)\right. \\
& \left.+\Lambda\left(\left\|D^{\beta}\left(x_{1}-x_{2}\right)\right\|\right)+\Lambda\left(\int_{0}^{s}|h(\xi)|\left\|x_{1}-x_{2}\right\| d \xi \mid\right)\right] d s \\
& +\int_{\lambda}^{\mu}|G(t, s)|\left[a_{1}(s)\left\|x_{1}-x_{2}\right\|+a_{2}(s)\left\|x_{1}^{\prime}-x_{2}^{\prime}\right\|\right. \\
& \left.+a_{3}(s)\left\|D^{\beta}\left(x_{1}-x_{2}\right)\right\|+a_{4}(s) \int_{0}^{s}|h(\xi)|\left\|x_{1}-x_{2}\right\| d \xi \mid\right] d s \\
& +\int_{\mu}^{\lambda}|G(t, s)|\left[\Lambda^{\prime}\left(\left\|x_{1}-x_{2}\right\|\right)+\Lambda^{\prime}\left(\left\|x_{1}^{\prime}-x_{2}^{\prime}\right\|\right)\right. \\
& \left.+\Lambda^{\prime}\left(\mid D^{\beta}\left\|x_{1}-x_{2}\right\|\right)+\Lambda^{\prime}\left(\int_{0}^{s}|h(\xi)|\left\|x_{1}-x_{2}\right\| d \xi \mid\right)\right] d s \\
& \leq \int_{0}^{\lambda}|G(t, s)|\left[\Lambda\left(\left\|x_{1}-x_{2}\right\|\right)+\Lambda\left(\left\|x_{1}^{\prime}-x_{2}^{\prime}\right\|\right)\right. \\
& \left.+\Lambda\left(\frac{\left\|x_{1}^{\prime}-x_{2}^{\prime}\right\|}{\Gamma(2-\beta)}\right)+\Lambda\left(m_{0}\left\|x_{1}-x_{2}\right\|\right)\right] d s \\
& +\int_{\lambda}^{\mu}|G(t, s)|\left[a_{1}(s)\left\|x_{1}-x_{2}\right\|+a_{2}(s)\left\|x_{1}^{\prime}-x_{2}^{\prime}\right\|\right. \\
& \left.+a_{3}(s) \frac{\left\|x_{1}^{\prime}-x_{2}^{\prime}\right\|}{\Gamma(2-\beta)}+a_{4}(s) m_{0}\left\|x_{1}-x_{2}\right\|\right] d s \\
& +\int_{\mu}^{\lambda}|G(t, s)|\left[\Lambda^{\prime}\left(\left\|x_{1}-x_{2}\right\|\right)+\Lambda^{\prime}\left(\left\|x_{1}^{\prime}-x_{2}^{\prime}\right\|\right)\right. \\
& \left.+\Lambda^{\prime}\left(\frac{\left\|x_{1}^{\prime}-x_{2}^{\prime}\right\|}{\Gamma(2-\beta)}\right)+\Lambda^{\prime}\left(m_{0}\left\|x_{1}-x_{2}\right\|\right)\right] d s \\
& \leq \int_{0}^{\lambda}|G(t, s)|\left[\Lambda\left(l\left\|x_{1}-x_{2}\right\|_{*}\right)+\Lambda\left(l\left\|x_{1}-x_{2}\right\|_{*}\right)\right. \\
& \left.+\Lambda\left(\frac{l\left\|x_{1}-x_{2}\right\|_{*}}{\Gamma(2-\beta)}\right)+\Lambda\left(m_{0} l\left\|x_{1}-x_{2}\right\|_{*}\right)\right] d s \\
& +\int_{\lambda}^{\mu}|G(t, s)|\left[a_{1}(s) l\left\|x_{1}-x_{2}\right\|_{*}+a_{2}(s) l\left\|x_{1}-x_{2}\right\|_{*}\right. \\
& \left.+a_{3}(s) \frac{l\left\|x_{1}-x_{2}\right\|_{*}}{\Gamma(2-\beta)}+a_{4}(s) m_{0} l\left\|x_{1}-x_{2}\right\|_{*}\right] d s \\
& +\int_{\mu}^{\lambda}|G(t, s)|\left[\Lambda^{\prime}\left(l\left\|x_{1}-x_{2}\right\|_{*}\right)+\Lambda^{\prime}\left(l\left\|x_{1}-x_{2}\right\|_{*}\right)\right. \\
& \left.+\Lambda^{\prime}\left(\frac{l\left\|x_{1}-x_{2}\right\|_{*}}{\Gamma(2-\beta)}\right)+\Lambda^{\prime}\left(m_{0} l\left\|x_{1}-x_{2}\right\|_{*}\right)\right] d s,
\end{aligned}
$$

where $m_{0}=\int_{0}^{1}|h(\xi)| d \xi$ and $l=\max \left\{1, \frac{1}{\Gamma(2-\beta)}, m_{0}, \theta_{0}+\theta_{1}\right\}$. On the other hand, $\lim _{z \rightarrow 0^{+}} \frac{\Lambda(z)}{z}=q$ and so for each $\epsilon>0$ there exists $0<\delta_{\Lambda}=\delta(\epsilon, \Lambda)$ such that $\left|\frac{\Lambda(z)}{z}-q\right|<\epsilon$ for all $0<z \leq \delta_{\Lambda}$. Thus, $0<z \leq \delta_{\Lambda}$ implies $\left|\frac{\Lambda(z)}{z}\right|-q \leq\left|\frac{\Lambda(z)}{z}-q\right|<\epsilon$. Hence, $|\Lambda(z)|<(\epsilon+q)|z|$. 
By choosing $0<z \leq \delta_{1}:=\min \left\{\delta_{\Lambda}, \epsilon\right\}$, we have

$$
|\Lambda(z)|<(\epsilon+q)|z|<(\epsilon+q) \epsilon
$$

For $\Lambda^{\prime}$ we have similar conclusion, that is,

$$
\left|\Lambda^{\prime}(z)\right|<\left(\epsilon+q^{\prime}\right) \epsilon
$$

for all $0<z \leq \delta_{1}:=\min \left\{\delta_{\Lambda^{\prime}}, \epsilon\right\}$. Let $\epsilon>0$ be given, $l\left\|x_{1}-x_{2}\right\|_{*}<\min \left\{\delta_{1}, \delta_{2}\right\}$ and $x_{1} \rightarrow x_{2}$. By using (2) and (3), we get $\Lambda\left(l\left\|x_{1}-x_{2}\right\|_{*}\right)<(\epsilon+q) \epsilon$ and $\Lambda^{\prime}\left(l\left\|x_{1}-x_{2}\right\|_{*}\right)<\left(\epsilon+q^{\prime}\right) \epsilon$. Now by using $\left(^{*}\right)$, we obtain

$$
\begin{aligned}
& \left|T_{x_{1}}(t)-T_{x_{2}}(t)\right| \\
& \leq 4(q+\epsilon) \epsilon \int_{0}^{\lambda}|G(t, s)| d s+\epsilon \int_{\lambda}^{\mu}\left[a_{1}(s)+\cdots+a_{4}(s)\right]|G(t, s)| d s \\
& \quad+4\left(q^{\prime}+\epsilon\right) \epsilon \int_{\mu}^{1}|G(t, s)| d s \leq 4(q+\epsilon) \epsilon \frac{2+\alpha+T_{0}}{\Gamma(\alpha)} \int_{0}^{\lambda}(1-s)^{\alpha-2} d s \\
& \quad+\frac{\epsilon\left(2+\alpha+T_{0}\right)}{\Gamma(\alpha)} \sum_{i=1}^{4} \int_{\lambda}^{\mu} a_{i}(s)(1-s)^{\alpha-2} d s+4\left(q^{\prime}+\epsilon\right) \epsilon \frac{2+\alpha+T_{0}}{\Gamma(\alpha)} \int_{\mu}^{1}(1-s)^{\alpha-2} d s \\
& =\epsilon \frac{2+\alpha+T_{0}}{\Gamma(\alpha)}\left[4(q+\epsilon) \cdot \frac{1}{\alpha-1}\left(1-(1-\lambda)^{\alpha-1}\right)\right. \\
& \left.\quad+\sum_{i=1}^{4}\left\|\hat{a}_{i}\right\|_{[\lambda, \mu]}+4\left(q^{\prime}+\epsilon\right) \cdot \frac{1}{\alpha-1}(1-\mu)^{\alpha-1}\right] .
\end{aligned}
$$

Hence,

$$
\begin{aligned}
\left\|T_{x_{1}}-T_{x_{2}}\right\| \leq & \epsilon \frac{2+\alpha+T_{0}}{\Gamma(\alpha)}\left[4(q+\epsilon) \cdot \frac{1}{\alpha-1}\left(1-(1-\lambda)^{\alpha-1}\right)\right. \\
& \left.+\sum_{i=1}^{4}\left\|\hat{a}_{i}\right\|_{[\lambda, \mu]}+4\left(q^{\prime}+\epsilon\right) \cdot \frac{1}{\alpha-1}(1-\mu)^{\alpha-1}\right] .
\end{aligned}
$$

In a similar way, we get

$$
\begin{aligned}
\left|T_{x_{1}}^{\prime}(t)-T_{x_{2}}^{\prime}(t)\right| & \\
\leq & \int_{0}^{1}\left|\frac{\partial G}{\partial t}(t, s)\right| \mid f\left(s, x_{1}(s), x_{1}^{\prime}(s), D^{\beta} x_{1}(s), \int_{0}^{s} h(\xi) x_{1}(\xi) d \xi\right) \\
& -f\left(s, x_{2}(s), x_{2}^{\prime}(s), D^{\beta} x_{2}(s), \int_{0}^{s} h(\xi) x_{2}(\xi) d \xi\right) \mid d s \\
\leq & \int_{0}^{\lambda}\left|\frac{\partial G}{\partial t}(t, s)\right| \mid f_{1}\left(s, x_{1}(s), x_{1}^{\prime}(s), D^{\beta} x_{1}(s), \int_{0}^{s} h(\xi) x_{1}(\xi) d \xi\right) \\
& -f_{1}\left(s, x_{2}(s), x_{2}^{\prime}(s), D^{\beta} x_{2}(s), \int_{0}^{s} h(\xi) x_{2}(\xi) d \xi\right) \mid d s
\end{aligned}
$$




$$
\begin{aligned}
& +\int_{\lambda}^{\mu}\left|\frac{\partial G}{\partial t}(t, s)\right| \mid f_{2}\left(s, x_{1}(s), x_{1}^{\prime}(s), D^{\beta} x_{1}(s), \int_{0}^{s} h(\xi) x_{1}(\xi) d \xi\right) \\
& -f_{2}\left(s, x_{2}(s), x_{2}^{\prime}(s), D^{\beta} x_{2}(s), \int_{0}^{s} h(\xi) x_{2}(\xi) d \xi\right) \mid d s \\
& +\int_{\mu}^{1}\left|\frac{\partial G}{\partial t}(t, s)\right| \mid f_{3}\left(s, x_{1}(s), x_{1}^{\prime}(s), D^{\beta} x_{1}(s), \int_{0}^{s} h(\xi) x_{1}(\xi) d \xi\right) \\
& -f_{3}\left(s, x_{2}(s), x_{2}^{\prime}(s), D^{\beta} x_{2}(s), \int_{0}^{s} h(\xi) x_{2}(\xi) d \xi\right) \mid d s \\
& \leq 4(q+\epsilon) \epsilon \int_{0}^{\lambda}\left|\frac{\partial G}{\partial t}(t, s)\right| d s+\epsilon \int_{\lambda}^{\mu}\left[a_{1}(s)+\cdots+a_{4}(s)\right]|G(t, s)| d s \\
& +4\left(q^{\prime}+\epsilon\right) \epsilon \int_{\mu}^{1}\left|\frac{\partial G}{\partial t}(t, s)\right| d s \leq 4(q+\epsilon) \frac{3 \epsilon \alpha}{\Gamma(\alpha)} \int_{0}^{\lambda}(1-s)^{\alpha-2} d s \\
& +\frac{3 \epsilon \alpha}{\Gamma(\alpha)} \sum_{i=1}^{4} \int_{\lambda}^{\mu} a_{i}(s)(1-s)^{\alpha-2} d s+4\left(q^{\prime}+\epsilon\right) \frac{3 \epsilon \alpha}{\Gamma(\alpha)} \int_{\mu}^{1}(1-s)^{\alpha-2} d s \\
& =\frac{3 \epsilon \alpha}{\Gamma(\alpha)}\left[4(q+\epsilon) \cdot \frac{1}{\alpha-1}\left(1-(1-\lambda)^{\alpha-1}\right)\right. \\
& \left.+\sum_{i=1}^{4}\left\|\hat{a}_{i}\right\|_{[\lambda, \mu]}+4\left(q^{\prime}+\epsilon\right) \cdot \frac{1}{\alpha-1}(1-\mu)^{\alpha-1}\right]
\end{aligned}
$$

and so

$$
\begin{aligned}
\left\|T_{x_{1}}^{\prime}-T_{x_{2}}^{\prime}\right\| \leq & \frac{3 \epsilon \alpha}{\Gamma(\alpha)}\left[4(q+\epsilon) \cdot \frac{1}{\alpha-1}\left(1-(1-\lambda)^{\alpha-1}\right)\right. \\
& \left.+\sum_{i=1}^{4}\left\|\hat{a}_{i}\right\|_{[\lambda, \mu]}+4\left(q^{\prime}+\epsilon\right) \cdot \frac{1}{\alpha-1}(1-\mu)^{\alpha-1}\right] .
\end{aligned}
$$

Hence,

$$
\begin{aligned}
\left\|T_{x_{1}}-T_{x_{2}}\right\|_{*} \leq & \epsilon\left[4(q+\epsilon) \cdot \frac{1}{\alpha-1}\left(1-(1-\lambda)^{\alpha-1}\right)+\sum_{i=1}^{4}\left\|\hat{a}_{i}\right\|_{[\lambda, \mu]}\right. \\
& \left.+4\left(q^{\prime}+\epsilon\right) \cdot \frac{1}{\alpha-1}(1-\mu)^{\alpha-1}\right] \max \left\{\frac{2+\alpha+T_{0}}{\Gamma(\alpha)}, \frac{3 \alpha}{\Gamma(\alpha)}\right\} .
\end{aligned}
$$

This implies that $\left\|T_{x_{1}}-T_{x_{2}}\right\|_{*} \rightarrow 0$ as $x_{1} \rightarrow x_{2}$. Hence, $T$ is continuous. Since $\lim _{z \rightarrow 0^{+}} \frac{\Lambda(z)}{z}=$ $q$ and $\Lambda$ is nondecreasing, for each $\epsilon>0$ there exists $\delta_{1}=\delta_{1}(\epsilon)>0$ such that $\frac{\Lambda(l z)}{l z}<q+\epsilon$ for all $z \in\left(0, \delta_{1}\right]$. Thus, $\Lambda(l z)<(q+\epsilon) l z$. By using similar reason, there exists $\delta_{2}(\epsilon)>0$ such that $\Lambda^{\prime}(l z)<\left(q^{\prime}+\epsilon\right) l z$ for all $z \in\left(0, \delta_{2}\right]$. Put $\delta=\delta(\epsilon):=\min \left\{\delta_{1}(\epsilon), \delta_{2}(\epsilon)\right\}$. Then $\Lambda(l z)<(q+\epsilon) l z$ and $\Lambda^{\prime}(l z)<\left(q^{\prime}+\epsilon\right) l z$ for all $z \in(0, \delta]$. In particular, $\Lambda(l \delta)<(q+\epsilon) l \delta$ and $\Lambda^{\prime}(l \delta)<\left(q^{\prime}+\epsilon\right) l \delta$. On other hand, we have $\frac{4 q}{\alpha-1}\left(1-(1-\lambda)^{\alpha-1}\right)+\sum_{i=1}^{4}\left\|\hat{a}_{i}\right\|_{[\lambda, \mu]}+\frac{4 q^{\prime}}{\alpha-1}(1-\mu)^{\alpha-1}<\frac{\Gamma(\alpha)}{l \theta_{0}}$. Choose $\epsilon_{0}>0$ such that $\frac{4\left(q+\epsilon_{0}\right)}{\alpha-1}\left(1-(1-\lambda)^{\alpha-1}\right)+\sum_{i=1}^{4}\left\|\hat{a}_{i}\right\|_{[\lambda, \mu]}+\frac{4\left(q^{\prime}+\epsilon_{0}\right)}{\alpha-1}(1-\mu)^{\alpha-1}<\frac{\Gamma(\alpha)}{l \theta_{0}}$ and put $\delta_{0}=\delta\left(\epsilon_{0}\right)$. Then $\Lambda\left(l \delta_{0}\right)<(q+\epsilon) l \delta_{0}$ and $\Lambda^{\prime}\left(l \delta_{0}\right)<\left(q^{\prime}+\epsilon\right) l \delta_{0}$. Now, assume that $E=\{x \in X$ : 
Baleanu et al. Advances in Difference Equations

(2019) 2019:153

Page 8 of 19

$\left.\|x\|_{*}<\delta_{0}\right\}, x \in E$ and $t \in[0,1]$. Then we have

$$
\begin{aligned}
& \left|T_{x}(t)\right| \leq \int_{0}^{1}|G(t, s)| f\left(s, x(s), x^{\prime}(s), D^{\beta} x(s), \int_{0}^{s} h(\xi) x(\xi) d \xi\right) \mid d s \\
& =\int_{0}^{\lambda}|G(t, s)| f_{1}\left(s, x(s), x^{\prime}(s), D^{\beta} x(s), \int_{0}^{s} h(\xi) x(\xi) d \xi\right) \mid d s \\
& +\int_{\lambda}^{\mu}|G(t, s)|\left|f_{2}\left(s, x(s), x^{\prime}(s), D^{\beta} x(s), \int_{0}^{s} h(\xi) x(\xi) d \xi\right)\right| d s \\
& +\int_{\mu}^{1}|G(t, s)|\left|f_{3}\left(s, x(s), x^{\prime}(s), D^{\beta} x(s), \int_{0}^{s} h(\xi) x(\xi) d \xi\right)\right| d s \\
& =\int_{0}^{\lambda}|G(t, s)|\left|f_{1}\left(s, x(s), x^{\prime}(s), D^{\beta} x(s), \int_{0}^{s} h(\xi) x(\xi) d \xi\right)-f_{1}(s, 0,0,0,0,0)\right| d s \\
& +\int_{\lambda}^{\mu}|G(t, s)|\left|f_{2}\left(s, x(s), x^{\prime}(s), D^{\beta} x(s), \int_{0}^{s} h(\xi) x(\xi) d \xi\right)-f_{2}(s, 0,0,0,0,0)\right| d s \\
& +\int_{\mu}^{1}|G(t, s)|\left|f_{3}\left(s, x(s), x^{\prime}(s), D^{\beta} x(s), \int_{0}^{s} h(\xi) x(\xi) d \xi\right)-f_{3}(s, 0,0,0,0,0)\right| d s \\
& \leq \int_{0}^{\lambda}|G(t, s)|\left[\Lambda(|x(s)|)+\Lambda\left(\left|x^{\prime}(s)\right|\right)+\Lambda\left(\left|D^{\beta} x(s)\right|\right)\right. \\
& \left.+\Lambda\left(\int_{0}^{s}|h(\xi)||x(\xi)| d \xi\right)\right] d s \\
& +\int_{\lambda}^{\mu}|G(t, s)|\left[a_{1}(s)|x(s)|+a_{2}(s)\left|x^{\prime}(s)\right|+a_{3}(s)\left|D^{\beta} x(s)\right|\right. \\
& \left.+a_{4}(s) \int_{0}^{s}|h(\xi)||x(\xi)| d \xi\right] d s \\
& +\int_{\mu}^{1}|G(t, s)|\left[\Lambda^{\prime}(|x(s)|)+\Lambda^{\prime}\left(\left|x^{\prime}(s)\right|\right)+\Lambda^{\prime}\left(\left|D^{\beta} x(s)\right|\right)\right. \\
& \left.+\Lambda^{\prime}\left(\int_{0}^{s}|h(\xi)||x(\xi)| d \xi\right)\right] d s \\
& \leq \int_{0}^{\lambda}|G(t, s)|\left[\Lambda(\|x\|)+\Lambda\left(\left\|x^{\prime}\right\|\right)+\Lambda\left(\frac{\left\|x^{\prime}\right\|}{\Gamma(2-\beta)}\right)+\Lambda\left(m_{0}\|x\|\right)\right] d s \\
& +\int_{\lambda}^{\mu}|G(t, s)|\left[a_{1}(s)\|x\|+a_{2}(s)\left\|x^{\prime}\right\|+a_{3}(s) \frac{\left\|x^{\prime}\right\|}{\Gamma(2-\beta)}+a_{4}(s) m_{0}\|x\|\right] d s \\
& +\int_{\mu}^{1}|G(t, s)|\left[\Lambda^{\prime}(\|x\|)+\Lambda^{\prime}\left(\left\|x^{\prime}\right\|\right)+\Lambda^{\prime}\left(\frac{\left\|x^{\prime}\right\|}{\Gamma(2-\beta)}\right)+\Lambda^{\prime}\left(m_{0}\|x\|\right)\right] d s \\
& \leq 4 \Lambda\left(l\|x\|_{*}\right) \int_{0}^{\lambda}|G(t, s)| d s \\
& +l\|x\|_{*} \int_{\lambda}^{\mu}|G(t, s)| \sum_{i=1}^{4} a_{i}(s) d s+4 \Lambda\left(l\|x\|_{*}\right) \int_{\mu}^{1}|G(t, s)| d s \\
& \leq 4 \Lambda\left(l \delta_{0}\right) \frac{2+\alpha+T_{0}}{\Gamma(\alpha)} \int_{0}^{\lambda}(1-s)^{\alpha-2} d s \\
& +l \delta_{0} \frac{2+\alpha+T_{0}}{\Gamma(\alpha)} \sum_{i=1}^{4} \int_{\lambda}^{\mu} a_{i}(s)(1-s)^{\alpha-2} d s
\end{aligned}
$$




$$
\begin{aligned}
& +4 \Lambda^{\prime}\left(l \delta_{0}\right) \frac{2+\alpha+T_{0}}{\Gamma(\alpha)} \int_{\mu}^{1}(1-s)^{\alpha-2} d s \\
\leq & 4\left(q+\epsilon_{0}\right) l \delta_{0} \frac{\left(2+\alpha+T_{0}\right)}{\Gamma(\alpha)} \cdot \frac{1}{\alpha-1}\left[1-(1-\lambda)^{\alpha-1}\right] \\
& +l \delta_{0} \frac{\left(2+\alpha+T_{0}\right)}{\Gamma(\alpha)} \sum_{i=1}^{4}\left\|\hat{a}_{i}\right\|_{[\lambda, \mu]} \\
& +4\left(q+\epsilon_{0}\right) l \delta_{0} \frac{\left(2+\alpha+T_{0}\right)}{\Gamma(\alpha)} \cdot \frac{1}{\alpha-1}\left[1-(1-\lambda)^{\alpha-1}\right] \\
= & \delta_{0} \frac{\left(2+\alpha+T_{0}\right) l}{\Gamma(\alpha)}\left[\frac{4\left(q+\epsilon_{0}\right)}{\alpha-1}\left(1-(1-\lambda)^{\alpha-1}\right)\right. \\
& +\sum_{i=1}^{4}\left\|\hat{a}_{i}\right\|_{[\lambda, \mu]}+\frac{4\left(q^{\prime}+\epsilon_{0}\right)}{\alpha-1}(1-\mu)^{\alpha-1}<\delta_{0} .
\end{aligned}
$$

Hence, $\|T x\| \leq \delta_{0}$. By using a similar method, we get

$$
\begin{aligned}
& \left|T_{x}^{\prime}(t)\right| \leq \int_{0}^{1}\left|\frac{\partial G}{\partial t}(t, s)\right| f\left(s, x(s), x^{\prime}(s), D^{\beta} x(s), \int_{0}^{s} h(\xi) x(\xi) d \xi\right) \mid d s \\
& =\int_{0}^{\lambda}\left|\frac{\partial G}{\partial t}(t, s)\right|\left|f_{1}\left(s, x(s), x^{\prime}(s), D^{\beta} x(s), \int_{0}^{s} h(\xi) x(\xi) d \xi\right)\right| d s \\
& +\int_{\lambda}^{\mu}\left|\frac{\partial G}{\partial t}(t, s)\right|\left|f_{2}\left(s, x(s), x^{\prime}(s), D^{\beta} x(s), \int_{0}^{s} h(\xi) x(\xi) d \xi\right)\right| d s \\
& +\int_{\mu}^{1}\left|\frac{\partial G}{\partial t}(t, s)\right|\left|f_{3}\left(s, x(s), x^{\prime}(s), D^{\beta} x(s), \int_{0}^{s} h(\xi) x(\xi) d \xi\right)\right| d s \\
& \leq \int_{0}^{\lambda}\left|\frac{\partial G}{\partial t}(t, s)\right|\left[\Lambda(|x(s)|)+\Lambda\left(\left|x^{\prime}(s)\right|\right)+\Lambda\left(\left|D^{\beta} x(s)\right|\right)\right. \\
& \left.+\Lambda\left(\int_{0}^{s}|h(\xi)||x(\xi)| d \xi\right)\right] d s \\
& +\int_{\lambda}^{\mu}\left|\frac{\partial G}{\partial t}(t, s)\right|\left[a_{1}(s)|x(s)|+a_{2}(s)\left|x^{\prime}(s)\right|+a_{3}(s)\left|D^{\beta} x(s)\right|\right. \\
& \left.+a_{4}(s) \int_{0}^{s}|h(\xi)||x(\xi)| d \xi\right] d s \\
& +\int_{\mu}^{1}\left|\frac{\partial G}{\partial t}(t, s)\right|\left[\Lambda^{\prime}(|x(s)|)+\Lambda^{\prime}\left(\left|x^{\prime}(s)\right|\right)+\Lambda^{\prime}\left(\left|D^{\beta} x(s)\right|\right)\right. \\
& \left.+\Lambda^{\prime}\left(\int_{0}^{s}|h(\xi)||x(\xi)| d \xi\right)\right] d s \\
& \leq \int_{0}^{\lambda}\left|\frac{\partial G}{\partial t}(t, s)\right|\left[\Lambda(\|x\|)+\Lambda\left(\left\|x^{\prime}\right\|\right)+\Lambda\left(\frac{\left\|x^{\prime}\right\|}{\Gamma(2-\beta)}\right)+\Lambda\left(m_{0}\|x\|\right)\right] d s \\
& +\int_{\lambda}^{\mu}\left|\frac{\partial G}{\partial t}(t, s)\right|\left[a_{1}(s)\|x\|+a_{2}(s)\left\|x^{\prime}\right\|+a_{3}(s) \frac{\left\|x^{\prime}\right\|}{\Gamma(2-\beta)}+a_{4}(s) m_{0}\|x\|\right] d s \\
& +\int_{\mu}^{1}\left|\frac{\partial G}{\partial t}(t, s)\right|\left[\Lambda^{\prime}(\|x\|)+\Lambda^{\prime}\left(\left\|x^{\prime}\right\|\right)+\Lambda^{\prime}\left(\frac{\left\|x^{\prime}\right\|}{\Gamma(2-\beta)}\right)+\Lambda^{\prime}\left(m_{0}\|x\|\right)\right] d s
\end{aligned}
$$




$$
\begin{aligned}
\leq & 4 \Lambda\left(l\|x\|_{*}\right) \int_{0}^{\lambda}\left|\frac{\partial G}{\partial t}(t, s)\right| d s+l\|x\|_{*} \int_{\lambda}^{\mu}\left|\frac{\partial G}{\partial t}(t, s)\right| \sum_{i=1}^{4} a_{i}(s) d s+4 \Lambda\left(l\|x\|_{*}\right) \\
& \times \int_{\mu}^{1}|G(t, s)| d s \leq 4 \Lambda\left(l \delta_{0}\right) \frac{3 \alpha}{\Gamma(\alpha)} \int_{0}^{\lambda}(1-s)^{\alpha-2} d s \\
& +l \delta_{0} \frac{3 \alpha}{\Gamma(\alpha)} \sum_{i=1}^{4} \int_{\lambda}^{\mu} a_{i}(s)(1-s)^{\alpha-2} d s+4 \Lambda^{\prime}\left(l \delta_{0}\right) \frac{3 \alpha}{\Gamma(\alpha)} \int_{\mu}^{1}(1-s)^{\alpha-2} d s \\
\leq & 4\left(q+\epsilon_{0}\right) l \delta_{0} \frac{3 \alpha}{\Gamma(\alpha)} \cdot \frac{1}{\alpha-1}\left[1-(1-\lambda)^{\alpha-1}\right]+l \delta_{0} \frac{3 \alpha}{\Gamma(\alpha)} \sum_{i=1}^{4}\left\|\hat{a}_{i}\right\|_{[\lambda, \mu]} \\
& +4\left(q+\epsilon_{0}\right) l \delta_{0} \frac{3 \alpha}{\Gamma(\alpha)} \cdot \frac{1}{\alpha-1}\left[1-(1-\lambda)^{\alpha-1}\right] \\
= & \delta_{0} \frac{3 \alpha l}{\Gamma(\alpha)}\left[\frac{4\left(q+\epsilon_{0}\right)}{\alpha-1}\left(1-(1-\lambda)^{\alpha-1}\right)+\sum_{i=1}^{4}\left\|\hat{a}_{i}\right\|_{[\lambda, \mu]}+\frac{4\left(q^{\prime}+\epsilon_{0}\right)}{\alpha-1}(1-\mu)^{\alpha-1}\right] \\
< & \delta_{0}
\end{aligned}
$$

for all $x \in E$ and $t \in[0,1]$. Hence, $\|T x\| \leq \delta_{0}$, and so $\|T x\|_{*} \leq \delta_{0}$. Thus, $T$ maps $E$ into $E$. It is easy to check that $T$ maps bounded sets into bounded sets. Assume that $t_{1}, t_{2} \in[0,1]$ and $x \in E$. Since $G(t, s)$ and $\frac{\partial G(t, s)}{\partial t}$ are continuous with respect to $t$, we get

$$
\begin{aligned}
\lim _{t_{2} \rightarrow t_{1}} T^{\prime} x\left(t_{2}\right) & =\lim _{t_{2} \rightarrow t_{1}} \int_{0}^{1}\left|\frac{\partial G}{\partial t}\left(t_{2}, s\right)\right|\left|f\left(s, x(s), x^{\prime}(s), D^{\beta} x(s), \int_{0}^{s} h(\xi) x(\xi) d \xi\right)\right| d s \\
& =\int_{0}^{1} \lim _{t_{2} \rightarrow t_{1}} \frac{\partial G}{\partial t}\left(t_{2}, s\right) f\left(s, x(s), x^{\prime}(s), D^{\beta} x(s), \int_{0}^{s} h(\xi) x(\xi) d \xi\right) d s \\
& =\int_{0}^{1} \frac{\partial G}{\partial t}\left(t_{1}, s\right) f\left(s, x(s), x^{\prime}(s), D^{\beta} x(s), \int_{0}^{s} h(\xi) x(\xi) d \xi\right) d s \\
& =T^{\prime} x\left(t_{1}\right) .
\end{aligned}
$$

Hence, $T$ is equi-continuous on $E$ and so $T: E \rightarrow E$ is completely continuous. Now by using Lemma 2, $T$ has a fixed point on $E$ and so the problem (1) has a solution.

Example 1 Consider the pointwise defined equation

$$
D^{\frac{7}{2}} x(t)+f\left(t, x(t), x^{\prime}(t), D^{\frac{1}{2}} x(t), \int_{0}^{t} s x(s) d s\right)=0
$$

with the boundary conditions in the last result, where

$$
f\left(t, x_{1}, x_{2}, x_{3}, x_{4}, x_{5}\right)= \begin{cases}t \sum_{i=1}^{4} x_{i}, & 0 \leq t<0.2 \\ d(t) \sum_{i=1}^{4} x_{i}, & 0.2 \leq t \leq 0.8 \\ t^{2} \sum_{i=1}^{4} x_{i}, & 0.8<t \leq 1\end{cases}
$$

and $d(t)=0$ whenever $t \in[0.1,0.8] \cap Q$ and $d(t)=0.1$ whenever $t \in[0.1,0.8] \cap Q^{c}$. Now, put $f_{1}\left(t, x_{1}, x_{2}, x_{3}, x_{4}, x_{5}\right)=\frac{1}{2} t \sum_{i=1}^{4} x_{i}, f_{2}\left(t, x_{1}, x_{2}, x_{3}, x_{4}, x_{5}\right)=d(t) \sum_{i=1}^{4} x_{i}$ and $f_{3}\left(t, x_{1}, x_{2}, x_{3}, x_{4}\right.$, 
$\left.x_{5}\right)=t^{2} \sum_{i=1}^{4} x_{i}$. Then we have $f_{1}(t, 0,0,0,0)=f_{2}(t, 0,0,0,0)=f_{3}(t, 0,0,0,0)=0$,

$$
\begin{aligned}
\left|f_{1}\left(t, x_{1}, x_{2}, x_{3}, x_{4}\right)-f_{1}\left(t, y_{1}, y_{2}, y_{3}, y_{4}\right)\right| & \leq t \sum_{i=1}^{4}|| x_{i}|-| y_{i}|| \\
& \leq t \sum_{i=1}^{4}\left|x_{i}-y_{i}\right| \leq 0.1 \sum_{i=1}^{4} \Lambda\left(\left|x_{i}-y_{i}\right|\right), \\
\left|f_{2}\left(t, x_{1}, x_{2}, x_{3}, x_{4}\right)-f_{2}\left(t, y_{1}, y_{2}, y_{3}, y_{4}\right)\right| & \leq d(t) \sum_{i=1}^{4}|| x_{i}|-| y_{i}|| \\
& \leq d(t) \sum_{i=1}^{4}\left|x_{i}-y_{i}\right|
\end{aligned}
$$

and

$$
\begin{aligned}
\left|f_{3}\left(t, x_{1}, x_{2}, x_{3}, x_{4}\right)-f_{3}\left(t, y_{1}, y_{2}, y_{3}, y_{4}\right)\right| & \leq \frac{1}{2} t \sum_{i=1}^{4}|| x_{i}|-| y_{i}|| \\
& \leq \frac{1}{2} t \sum_{i=1}^{4}\left|x_{i}-y_{i}\right| \leq \frac{1}{2} \sum_{i=1}^{4} \Lambda\left(\left|x_{i}-y_{i}\right|\right),
\end{aligned}
$$

where $\Lambda(x)=|x|$ and $\Lambda^{\prime}(x)=\frac{1}{2}|x|$. Hence, $\lim _{z \rightarrow 0^{+}} \frac{\Lambda^{\prime}(z)}{z}=0.1:=q, \lim _{z \rightarrow 0^{+}} \frac{\Lambda^{\prime}(z)}{z}=\frac{1}{2}:=q^{\prime}$, $\hat{a}_{i}=\hat{d} \in L^{1}[0.1,0.8], \sum_{i=1}^{4}\left\|\hat{a}_{i}\right\|_{[\lambda, \mu]}<0.092$ and

$$
\begin{aligned}
& {\left[\frac{4 q\left(1-(1-\lambda)^{\alpha-1}\right)}{\alpha-1}+\sum_{i=1}^{4}\left\|\hat{a}_{i}\right\|+\frac{4 q^{\prime}}{\alpha-1}(1-\mu)^{\alpha-1}\right]} \\
& \quad<\left[\frac{4 \times 0.1\left(1-(1-0.1)^{\frac{5}{2}}\right.}{\frac{5}{2}}+0.092+\frac{4 \times 0.5(1-0.9)^{\frac{5}{2}}}{\frac{5}{2}}\right]<\frac{\Gamma(\alpha)}{3 l \alpha} .
\end{aligned}
$$

Now by using Theorem 5 , the problem has a solution.

Now, we present our second result by using different conditions.

Theorem 6 Suppose that $f=\left[f_{1}, f_{2}, f_{3}, \lambda, \mu\right], f$ is nonnegative on $[0,1]$ and there exist nonnegative functions $a_{1}, a_{2}, a_{3}, a_{4}:[0, \lambda] \rightarrow \mathbb{R}^{+}$, maps $b_{1}, \ldots, b_{k_{0}}:[\lambda, \mu] \rightarrow \mathbb{R}^{+}$for some $k_{0} \geq 1$, and functions $c_{1}, c_{2}, c_{3}, c_{4}:[\mu, 1] \rightarrow \mathbb{R}^{+}$such that $\hat{a}_{i} \in L^{1}[0, \lambda], \hat{b}_{j} \in L^{1}[\lambda, \mu], \hat{c}_{i} \in L^{1}[\mu, 1]$ and $\hat{a}_{1}(s)=(1-s)^{\alpha-2} a_{i}(s)$. Assuming that there are nonnegative and nondecreasing functions $\phi_{i}, \Phi_{i}: \mathbb{R}^{+} \rightarrow \mathbb{R}^{+}$and $H_{j}: \mathbb{R}_{+}{ }^{4} \rightarrow \mathbb{R}_{+}$such that $\lim _{z \rightarrow 0^{+}} \frac{\phi_{i}(z)}{z^{\mu_{i}}}:=l_{\mu_{i}}<\infty, \lim _{z \rightarrow 0^{+}} \frac{\Phi_{i}(z)}{z^{\gamma_{i}}}:=$ $l_{\gamma_{i}}<\infty$ and $\lim _{z \rightarrow 0^{+}} \frac{H_{j}(z, z, z, z)}{z^{m}}:=q_{j}<\infty$ for some $\mu_{i}, \gamma_{i}, m \in[1, \infty)$ and $H_{j}$ are nonnegative and nondecreasing with respect to all their components $\left(1 \leq i \leq 4,1 \leq j \leq k_{0}\right)$,

$$
\begin{aligned}
& \left|f_{1}\left(t, x_{1}, \ldots, x_{4}\right)-f_{1}\left(t, y_{1}, \ldots, y_{4}\right)\right| \leq \sum_{i=1}^{4} a_{i}(t) \phi_{i}\left(\left|x_{i}-y_{i}\right|\right) \\
& \left|f_{2}\left(t, x_{1}, \ldots, x_{4}\right)-f_{2}\left(t, y_{1}, \ldots, y_{4}\right)\right| \leq \sum_{i=1}^{5} b_{j}(t) H_{j}\left(\left|x_{1}-y_{1}\right|, \ldots,\left|x_{4}-y_{4}\right|\right)
\end{aligned}
$$


and $\left|f_{3}\left(t, x_{1}, \ldots, x_{4}\right)-f_{3}\left(t, y_{1}, \ldots, y_{4}\right)\right| \leq \sum_{i=1}^{4} c_{i}(t) \Phi_{i}\left(\left|x_{i}-y_{i}\right|\right)$. Suppose that $\left|f_{2}\left(t, x_{1}, \ldots, x_{4}\right)\right| \leq$ $\Theta(t) \Lambda\left(x_{1}, \ldots, x_{4}\right)$, where $\Lambda$ are nonnegative and nondecreasing with respect to all their components, $\lim _{x \rightarrow 0^{+}} \frac{\Lambda(x, x, x, x)}{x}:=P_{2},<\infty, \hat{\Theta} \in L^{1}[\lambda, \mu], \lim _{\max }\left|x_{i}\right| \rightarrow 0 \frac{\left|f_{1}\left(t, x_{1}, \ldots, x_{4}\right)\right|}{\max \left|x_{i}\right|}=P_{1}(t)$ and

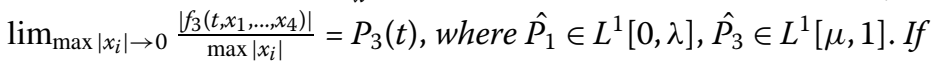

$$
\begin{aligned}
& \max \left\{\frac{3 \alpha}{\Gamma(\alpha)}, \frac{2+\alpha+T_{0}}{\Gamma(\alpha)}\right\} \max \left\{\sum_{i=1}^{5}\left\|\hat{a}_{i}\right\|_{[0, \lambda]}\left(l_{\mu_{i}}\right)+\sum_{j=1}^{k_{0}}\left\|\hat{b}_{j}\right\|_{[\lambda, \mu]} q_{j}\right. \\
& \left.\left.\quad+\sum_{i=1}^{4}\left\|\hat{c}_{i}\right\|_{[\mu, 1]}\right]\left(l_{\gamma_{i}}\right), \max \left\{1, \frac{1}{\Gamma(2-\beta)}, m_{0}\right\}\left[\left\|\hat{P}_{1}\right\|_{[0, \lambda]}+P_{2}\|\Theta\|_{[\lambda, \mu]}+\left\|\hat{P}_{3}\right\|_{[\mu, 1]}\right]\right\}<1,
\end{aligned}
$$

then the pointwise defined equation (1) with boundary conditions has a solution.

Proof Let $x, y \in X$ and $t \in[0,1]$. Then we have

$$
\begin{aligned}
\mid F_{x}(t) & -F_{y}(t) \mid \\
\leq & \int_{0}^{\lambda}|G(t, s)| f_{1}\left(s, x(s), x^{\prime}(s), D^{\beta} x(s), \int_{0}^{s} h(\xi) x(\xi) d \xi\right) \\
& -f_{1}\left(s, y(s), y^{\prime}(s), D^{\beta} y(s), \int_{0}^{s} h(\xi) y(\xi) d \xi\right) \mid d s \\
& +\int_{\lambda}^{\mu}|G(t, s)| f_{2}\left(s, x(s), x^{\prime}(s), D^{\beta} x(s), \int_{0}^{s} h(\xi) x(\xi) d \xi\right) \\
& -f_{2}\left(s, y(s), y^{\prime}(s), D^{\beta} y(s), \int_{0}^{s} h(\xi) y(\xi) d \xi\right) \mid d s \\
& +\int_{\mu}^{1}|G(t, s)| \mid f_{3}\left(s, x(s), x^{\prime}(s), D^{\beta} x(s), \int_{0}^{s} h(\xi) x(\xi) d \xi\right) \\
& -f_{3}\left(s, y(s), y^{\prime}(s), D^{\beta} y(s), \int_{0}^{s} h(\xi) y(\xi) d \xi\right) \mid d s \\
\leq & \int_{0}^{\lambda}|G(t, s)|\left[a_{1}(s) \phi(|x(s)-y(s)|)+a_{2}(s) \phi\left(\left|x^{\prime}(s)-y^{\prime}(s)\right|\right)\right. \\
& +a_{3}(s) \phi\left(\left|D^{\beta} x(s)-D^{\beta} y(s)\right|\right)+a_{4}(s) \phi\left(\left|\int_{0}^{s} h(\xi)(x(\xi)-y(\xi)) d \xi\right|\right) d s \\
& +\int_{\lambda}^{\mu}|G(t, s)| \sum_{i=1}^{k_{0}} b_{i}(s) H_{i}\left(|x(s)-y(s)|,\left|x^{\prime}(s)-y^{\prime}(s)\right|,\right. \\
& +c_{3}(s) \Phi\left(\left|D^{\beta} x(s)-D^{\beta} y(s)\right|\right)+c_{4}(s) \Phi\left(\left|\int_{0}^{s} h(\xi)(x(\xi)-y(\xi)) d \xi\right|\right] d s \\
& +D^{\beta} x(s)-D^{\beta} y(s)|,| \int_{0}^{s} h(\xi)(x(\xi)-y(\xi) d \xi) \mid d s \\
& \Gamma(\alpha) \int_{0}^{1}(1-s)^{\alpha-2}\left[a_{1}(s) \phi(\|x-y\|)+a_{2}(s) \phi\left(\left\|x^{\prime}-y^{\prime}\right\|\right)\right] \\
& +c_{1}(s) \Phi(|x(s)-y(s)|)+c_{2}(s) \Phi\left(\left|x^{\prime}(s)-y^{\prime}(s)\right|\right) \\
& \\
&
\end{aligned}
$$




$$
\begin{aligned}
& +a_{3}(s) \phi\left(\frac{\left\|x^{\prime}-y^{\prime}\right\|}{\Gamma(2-\beta)}\right)+a_{4}(s) \phi\left(m_{0}\|x-y\|\right) d s \\
& +\frac{2+\alpha+T_{0}}{\Gamma(\alpha)} \int_{\lambda}^{\mu}(1-s)^{\alpha-2} \\
& +\sum_{i=1}^{k_{0}} b_{i}(s) H_{i}\left(\|x-y\|,\left\|x^{\prime}-y^{\prime}\right\|, \frac{\left\|x^{\prime}-y^{\prime}\right\|}{\Gamma(2-\beta)}, m_{0}\|x-y\|\right) d s \\
& +\frac{2+\alpha+T_{0}}{\Gamma(\alpha)} \int_{\mu}^{1}(1-s)^{\alpha-2}\left[c_{1}(s) \Phi(\|x-y\|)+c_{2}(s) \Phi\left(\left\|x^{\prime}-y^{\prime}\right\|\right)\right. \\
& \left.+c_{3}(s) \Phi\left(\frac{\left\|x^{\prime}-y^{\prime}\right\|}{\Gamma(2-\beta)}\right)+c_{4}(s) \Phi\left(m_{0}\|x-y\|\right)\right] d s \\
& \leq \frac{2+\alpha+T_{0}}{\Gamma(\alpha)}\left[\sum_{i=1}^{4} \phi\left(l\|x-y\|_{*}\right) \int_{0}^{\lambda}(1-s)^{\alpha-2} a_{i}(s) d s\right. \\
& +\sum_{i=1}^{k_{0}} H_{i}\left(l\|x-y\|_{*}, \ldots, l\|x-y\|_{*}\right) \int_{\lambda}^{\mu}(1-s)^{\alpha-2} b_{i}(s) d s \\
& \left.+\sum_{i=1}^{4} \Phi\left(l\|x-y\|_{*}\right) \int_{\mu}^{1}(1-s)^{\alpha-2} c_{i}(s) d s\right] \\
& =\frac{2+\alpha+T_{0}}{\Gamma(\alpha)}\left[\sum_{i=1}^{5} \phi\left(l\|x-y\|_{*}\right)\left\|\hat{a}_{i}\right\|_{[0, \lambda]}\right. \\
& \left.+\sum_{i=1}^{k_{0}} H_{i}\left(l\|x-y\|_{*}, \ldots, l\|x-y\|_{*}\right)\left\|\hat{b}_{i}\right\|_{[\lambda, \mu]}+\sum_{i=1}^{4} \Phi\left(l\|x-y\|_{*}\right)\left\|\hat{c}_{i}\right\|_{[\mu, 1]}\right] .
\end{aligned}
$$

On the other hand, $\lim _{z \rightarrow 0^{+}} \frac{\phi_{i}(z)}{z^{\mu_{i}}}=l_{\mu_{i}}$ for $1 \leq i \leq 4$. This implies that for each $\epsilon>0$ there exists $0<\delta_{i}=\delta_{i}(\epsilon)<\epsilon$ such that $\frac{\phi_{i}(z)}{z^{\mu_{i}}}<l_{\mu_{i}}+\epsilon$ for all $z \in\left(0, \delta_{i}\right]$. Hence, $\phi_{i}\left(\delta_{i}\right)<\left(l_{\mu_{i}}+\epsilon\right) \delta_{i}^{\mu_{i}}<$ $\left(l_{\mu_{i}}+\epsilon\right) \epsilon^{\mu_{i}}$. By using a similar method, we conclude that there exists $0<\delta_{i}^{\prime}=\delta_{i}^{\prime}(\epsilon)<\epsilon$ such that $\Phi_{i}\left(\delta_{i}^{\prime}\right)<\left(l_{\gamma_{i}}+\epsilon\right)\left(\delta_{i}^{\prime}\right)^{\gamma_{i}}<\left(l_{\gamma_{i}}+\epsilon\right) \epsilon^{\gamma_{i}}$. Also, we have $\lim _{z \rightarrow 0^{+}} \frac{H_{j}(z, z, z, z)}{z^{m}}=q_{j}$ for $1 \leq j \leq k_{0}$ and so there exists $0<\delta_{q_{j}}<\epsilon$ such that $\frac{H_{j}(z, z, z, z)}{z^{m}}<q_{j}+\epsilon$ for all $z \in\left(0, \delta_{q_{j}}\right]$ and $1 \leq j \leq k_{0}$. Hence, $H_{j}(z, z, z, z)<\left(q_{j}+\epsilon\right) z^{m}$ for all $z \in\left(0, \delta_{q_{j}}\right]$ and so $H_{j}\left(\delta_{q_{j}}, \delta_{q_{j}}, \delta_{q_{j}}, \delta_{q_{j}}\right)<\left(q_{j}+\epsilon\right) \delta_{q_{j}}^{m}<\left(q_{j}+\epsilon\right) \epsilon^{m}$. Let $x \rightarrow y$ in $X$. If $l\|x-y\|_{*}<\delta:=\min \left\{\delta_{1}, \ldots, \delta_{4}, \delta_{1}^{\prime}, \ldots, \delta_{4}^{\prime}, \delta_{q_{1}}, \ldots, \delta_{q_{k_{0}}}\right\}$, then $\phi_{i}(\delta)<\phi_{i}\left(\delta_{i}\right)<$ $\left(l_{\mu_{i}}+\epsilon\right) \epsilon^{\mu_{i}}, \Phi_{i}(\delta)<\Phi_{i}\left(\delta_{i}^{\prime}\right)<\left(l_{\gamma_{i}}+\epsilon\right) \epsilon^{\gamma_{i}}$ and $H_{j}(\delta, \ldots, \delta)<H_{j}\left(\delta_{q_{j}}, \ldots, \delta_{q_{j}}\right)<\left(q_{j}+\epsilon\right) \epsilon^{m}$ for $1 \leq i \leq 4$ and $1 \leq j \leq k_{0}$. If $l\|x-y\|_{*}<\delta$, then $\left|F_{x}(t)-F_{y}(t)\right| \leq \frac{2+\alpha+T_{0}}{\Gamma(\alpha)}\left[\sum_{i=1}^{5}\left\|\hat{a}_{i}\right\|_{[0, \lambda]}\left(l_{\mu_{i}}+\right.\right.$ $\left.\epsilon) \epsilon^{\mu_{i}}+\sum_{j=1}^{k_{0}}\left\|\hat{b}_{j}\right\|_{[\lambda, \mu]}\left(q_{j}+\epsilon\right) \epsilon^{m}+\sum_{i=1}^{4}\left\|\hat{c}_{i}\right\|_{[\mu, 1]}\right]\left(l_{\gamma_{i}}+\epsilon\right) \epsilon^{\left.\gamma_{i}\right]}$ and so $\mid F_{x}-F_{y} \| \leq \frac{2+\alpha+T_{0}}{\Gamma(\alpha)} \times$ $\left.\left[\sum_{i=1}^{5}\left\|\hat{a}_{i}\right\|_{[0, \lambda]}\left(l_{\mu_{i}}+\epsilon\right) \epsilon^{\mu_{i}}+\sum_{j=1}^{k_{0}}\left\|\hat{b}_{j}\right\|_{[\lambda, \mu]}\left(q_{j}+\epsilon\right) \epsilon^{m}+\sum_{i=1}^{4}\left\|\hat{c}_{i}\right\|_{[\mu, 1]}\right]\left(l_{\gamma_{i}}+\epsilon\right) \epsilon^{\gamma_{i}}\right]$. By a similar way, we get

$$
\begin{aligned}
\left\|F_{x}^{\prime}-F_{y}^{\prime}\right\| \leq & \frac{3 \alpha}{\Gamma(\alpha)}\left[\sum_{i=1}^{5}\left\|\hat{a}_{i}\right\|_{[0, \lambda]}\left(l_{\mu_{i}}+\epsilon\right) \epsilon^{\mu_{i}}+\sum_{j=1}^{k_{0}}\left\|\hat{b}_{j}\right\|_{[\lambda, \mu]}\left(q_{j}+\epsilon\right) \epsilon^{m}\right. \\
& \left.+\sum_{i=1}^{4}\left\|\hat{c}_{i}\right\|_{[\mu, 1]}\right]\left(l_{\gamma_{i}}+\epsilon\right) \epsilon^{\left.\gamma_{i}\right]}
\end{aligned}
$$

and so $\left\|F_{x}-F_{y}\right\|_{*} \leq \max \left\{\frac{3 \alpha}{\Gamma(\alpha)}, \frac{2+\alpha+T_{0}}{\Gamma(\alpha)}\right\}\left[\sum_{i=1}^{5}\left\|\hat{a}_{i}\right\|_{[0, \lambda]}\left(l_{\mu_{i}}+\epsilon\right) \epsilon^{\mu_{i}}+\sum_{j=1}^{k_{0}}\left\|\hat{b}_{j}\right\|_{[\lambda, \mu]}\left(q_{j}+\epsilon\right) \epsilon^{m}+\right.$ $\left.\left.\sum_{i=1}^{4}\left\|\hat{c}_{i}\right\|_{[\mu, 1]}\right]\left(l_{\gamma_{i}}+\epsilon\right) \epsilon^{\gamma_{i}}\right]$. Since $\epsilon>0$ was arbitrary, we conclude that $\left\|F_{x}-F_{y}\right\|_{*} \rightarrow 0$ as $x \rightarrow$ 
$y$. This implies that $F$ is continuous on $X$. Since $\lim _{x \rightarrow 0^{+}} \frac{\Lambda(x, x, x, x)}{x}=P_{2}, \lim _{x \rightarrow 0^{+}} \frac{\Lambda(l x, l x, l x, l x)}{l x}=$ $P_{2}$, where $l=\max \left\{1, \frac{1}{\Gamma(2-\beta)}, m_{0}\right\}$. Thus for each $\epsilon>0$ there exists $\delta_{1}=\delta_{1}(\epsilon)$ such that $\frac{\Lambda(l x, l x, l x, l x)}{l x}<P_{2}+\epsilon$ for all $x \in\left(0, \delta_{1}\right]$. Hence,

$$
\Lambda(l x, l x, l x, l x)<\left(P_{2}+\epsilon\right) l x
$$

for $x \in\left(0, \delta_{1}\right]$. Also, $\lim _{\left|x_{i}\right| \rightarrow 0} \frac{\left|f_{1}\left(t, x_{1}, \ldots, x_{4}\right)\right|}{\min \left|x_{i}\right|}=P_{1}(t)$. Thus, there exists $\delta_{2}=\delta_{2}(\epsilon)$ such that

$$
\left|f_{1}\left(t, x_{1}, \ldots, x_{4}\right)\right|<\left(P_{1}(t)+\epsilon\right) \min \left|x_{i}\right|
$$

for all $t \in[0,1]$ and $\left|x_{i}\right| \in\left(0, \delta_{2}\right]$ for $1 \leq i \leq 4$. Similarly, there exists $\delta_{3}=\delta_{3}(\epsilon)$ such that

$$
\left|f_{3}\left(t, x_{1}, \ldots, x_{4}\right)\right|<\left(P_{3}(t)+\epsilon\right) \min \left|x_{i}\right|
$$

for all $t \in[0,1]$ and $\left|x_{i}\right| \in\left(0, \delta_{3}\right]$ for $1 \leq i \leq 4$. Since $\left\|\hat{P}_{1}\right\|_{[0, \lambda]}+P_{2}\|\Theta\|_{[\lambda, \mu]}+\left\|\hat{P}_{3}\right\|_{[\mu, 1]}<\frac{\Gamma(\alpha)}{l \theta_{0}}$, we can choose $\epsilon_{0}>0$ such that $\left\|\hat{P}_{1}\right\|_{[0, \lambda]}+\frac{\epsilon_{0}}{\alpha-1}\left(1-(1-\lambda)^{\alpha-1}\right)+\left(P_{2}+\epsilon_{0}\right)\|\Theta\|_{[\lambda, \mu]}+\left\|\hat{P}_{3}\right\|_{[\mu, 1]}+$ $\frac{\epsilon_{0}}{\alpha-1}(1-\mu)^{\alpha-1}<\frac{\Gamma(\alpha)}{l \theta_{0}}$. Since

$$
\left.\max \left\{\frac{3 \alpha}{\Gamma(\alpha)}, \frac{2+\alpha+T_{0}}{\Gamma(\alpha)}\right\}\left[\sum_{i=1}^{5}\left\|\hat{a}_{i}\right\|_{[0, \lambda]}\left(l_{\mu_{i}}\right)+\sum_{j=1}^{k_{0}}\left\|\hat{b}_{j}\right\|_{[\lambda, \mu]} q_{j}+\sum_{i=1}^{4}\left\|\hat{c}_{i}\right\|_{[\mu, 1]}\right]\left(l_{\gamma_{i}}\right)\right]<1
$$

pick $\epsilon_{1} \in(0,1)$ such that

$$
\begin{aligned}
& \max \left\{\frac{3 \alpha}{\Gamma(\alpha)}, \frac{2+\alpha+T_{0}}{\Gamma(\alpha)}\right\}\left[\sum_{i=1}^{5}\left\|\hat{a}_{i}\right\|_{[0, \lambda]}\left(l_{\mu_{i}}+\epsilon_{1}\right)\right. \\
& \left.\left.+\sum_{j=1}^{k_{0}}\left\|\hat{b}_{j}\right\|_{[\lambda, \mu]}\left(q_{j}+\epsilon_{1}\right)+\sum_{i=1}^{4}\left\|\hat{c}_{i}\right\|_{[\mu, 1]}\right]\left(l_{\gamma_{i}}+\epsilon_{1}\right)\right]<1 .
\end{aligned}
$$

Let $r_{0}=\min \left\{\delta_{1}\left(\epsilon_{0}\right), \delta_{2}\left(\epsilon_{0}\right), \delta_{3}\left(\epsilon_{0}\right), \frac{\epsilon_{1}}{2}\right\}$, and $C=\left\{x \in X:\|x\|_{*}<r_{0}\right\}$. Define the map $\alpha$ on $X \times$ $X$ by

$$
\alpha(x, y)= \begin{cases}1, & x, y \in C \\ 0, & \text { otherwise }\end{cases}
$$

Let $x, y \in X$ and $\alpha(x, y) \geq 1$. Then $x, y \in C$ and so

$$
\begin{aligned}
\left|F_{x}(t)\right| \leq & \int_{0}^{\lambda}|G(t, s)| f_{1}\left(s, x(s), x^{\prime}(s), D^{\beta} x(s), \int_{0}^{s} h(\xi) x(\xi) d \xi\right) \mid d s \\
& +\int_{\lambda}^{\mu}|G(t, s)|\left|f_{2}\left(s, x(s), x^{\prime}(s), D^{\beta} x(s), \int_{0}^{s} h(\xi) x(\xi) d \xi\right)\right| d s \\
& +\int_{\mu}^{1}|G(t, s)|\left|f_{3}\left(s, x(s), x^{\prime}(s), D^{\beta} x(s), \int_{0}^{s} h(\xi) x(\xi) d \xi\right)\right| d s \\
\leq & \frac{2+\alpha+T_{0}}{\Gamma(\alpha)}\left[\int_{0}^{\lambda}(1-s)^{\alpha-2}\left|f_{1}\left(s, x(s), x^{\prime}(s), D^{\beta} x(s), \int_{0}^{s} h(\xi) x(\xi) d \xi\right)\right| d s\right.
\end{aligned}
$$




$$
\begin{aligned}
& +\int_{\lambda}^{\mu}(1-s)^{\alpha-2}\left|f_{2}\left(s, x(s), x^{\prime}(s), D^{\beta} x(s), \int_{0}^{s} h(\xi) x(\xi) d \xi\right)\right| d s \\
& \left.+\int_{\mu}^{1}(1-s)^{\alpha-2}\left|3_{2}\left(s, x(s), x^{\prime}(s), D^{\beta} x(s), \int_{0}^{s} h(\xi) x(\xi) d \xi\right)\right| d s\right] \\
& \leq \frac{2+\alpha+T_{0}}{\Gamma(\alpha)}\left[\int_{0}^{\lambda}(1-s)^{\alpha-2}\left|f_{1}\left(s, x(s), x^{\prime}(s), D^{\beta} x(s), \int_{0}^{s} h(\xi) x(\xi) d \xi\right)\right| d s\right. \\
& +\int_{\lambda}^{\mu}(1-s)^{\alpha-2} \Theta(s) \Lambda\left(x(s), x^{\prime}(s), D^{\beta} x(s), \int_{0}^{s} h(\xi) x(\xi) d \xi\right) d s \\
& \left.+\int_{\mu}^{1}(1-s)^{\alpha-2}\left|3_{2}\left(s, x(s), x^{\prime}(s), D^{\beta} x(s), \int_{0}^{s} h(\xi) x(\xi) d \xi\right)\right| d s\right] \\
& \leq \frac{2+\alpha+T_{0}}{\Gamma(\alpha)}\left[\int_{0}^{\lambda}(1-s)^{\alpha-2}\left|f_{1}\left(s, x(s), x^{\prime}(s), D^{\beta} x(s), \int_{0}^{s} h(\xi) x(\xi) d \xi\right)\right| d s\right. \\
& +\int_{\lambda}^{\mu}(1-s)^{\alpha-2} \Theta(s) \Lambda\left(\|x\|,\left\|x^{\prime}\right\|, \frac{\left\|x^{\prime}\right\|}{\Gamma(2-\beta)}, m_{0}\|x\|\right) d s \\
& \left.+\int_{\mu}^{1}(1-s)^{\alpha-2}\left|3_{2}\left(s, x(s), x^{\prime}(s), D^{\beta} x(s), \int_{0}^{s} h(\xi) x(\xi) d \xi\right)\right| d s\right] \\
& \leq \frac{2+\alpha+T_{0}}{\Gamma(\alpha)}\left[\int_{0}^{\lambda}(1-s)^{\alpha-2}\left|f_{1}\left(s, x(s), x^{\prime}(s), D^{\beta} x(s), \int_{0}^{s} h(\xi) x(\xi) d \xi\right)\right| d s\right. \\
& +\int_{\lambda}^{\mu}(1-s)^{\alpha-2} \Theta(s) \Lambda\left(l\|x\|_{*}, l\|x\|_{*}, l\|x\|_{*}, l\|x\|_{*}\right) d s \\
& \left.+\int_{\mu}^{1}(1-s)^{\alpha-2}\left|3_{2}\left(s, x(s), x^{\prime}(s), D^{\beta} x(s), \int_{0}^{s} h(\xi) x(\xi) d \xi\right)\right| d s\right]
\end{aligned}
$$

for all $t \in[0,1]$. Since $\|x\|_{*}<r_{0}, x \in\left[0, \min \left\{\delta_{1}, \delta_{2}, \delta_{3}\right\}\right)$ and so by using (4), (5) and (6) we conclude that

$$
\begin{aligned}
\left|F_{x}(t)\right| \leq & \frac{2+\alpha+T_{0}}{\Gamma(\alpha)} \\
& \times\left[\int_{0}^{\lambda}(1-s)^{\alpha-2}\left(P_{1}(s)+\epsilon\right) \min \left\{x(s), x^{\prime}(s), D^{\beta} x(s), \int_{0}^{s} h(\xi) x(\xi) d \xi\right\} d s\right. \\
& +\left(P_{2}+\epsilon_{0}\right) l\|x\|_{*} \int_{\lambda}^{\mu}(1-s)^{\alpha-2} \Theta(s) d s \\
& \left.+\int_{\mu}^{1}(1-s)^{\alpha-2} \mid\left(P_{3}(s)+\epsilon_{0}\right) \min \left\{x(s), x^{\prime}(s), D^{\beta} x(s), \int_{0}^{s} h(\xi) x(\xi) d \xi\right\} d s\right] \\
\leq & \frac{2+\alpha+T_{0}}{\Gamma(\alpha)}\left[\int_{0}^{\lambda}(1-s)^{\alpha-2}\left(P_{1}(s)+\epsilon\right) \min \left\{\|x\|,\left\|x^{\prime}\right\|, \frac{\left\|x^{\prime}\right\|}{\Gamma(2-\beta)}, m_{0}\|x\|\right\} d s\right. \\
& \left.+\left(P_{2}+\epsilon_{0}\right) l\|x\|_{*}\|\hat{\Theta}\|_{[\lambda, \mu]}\right] \\
& \left.+\int_{\mu}^{1}(1-s)^{\alpha-2} \mid\left(P_{3}(s)+\epsilon_{0}\right) \min \left\{\|x\|,\left\|x^{\prime}\right\|, \frac{\left\|x^{\prime}\right\|}{\Gamma(2-\beta)}, m_{0}\|x\|\right\} d s\right] \\
\leq & \frac{2+\alpha+T_{0}}{\Gamma(\alpha)}\left[l\|x\|_{*} \int_{0}^{\lambda}(1-s)^{\alpha-2}\left(P_{1}(s)+\epsilon_{0}\right) d s+\left(P_{2}+\epsilon\right) l\|x\|_{*}\|\hat{\Theta}\|_{[\lambda, \mu]}\right. \\
& \left.+l\|x\|_{*} \int_{\mu}^{1}(1-s)^{\alpha-2}\left(P_{3}(s)+\epsilon_{0}\right) d s\right]
\end{aligned}
$$




$$
\begin{aligned}
= & \frac{2+\alpha+T_{0}}{\Gamma(\alpha)} l\|x\|_{*}\left[\int_{0}^{\lambda}(1-s)^{\alpha-2} P_{1}(s) d s+\epsilon_{0} \int_{0}^{\lambda}(1-s)^{\alpha-2} d s\right. \\
& \left.+\left(P_{2}+\epsilon_{0}\right)\|\hat{\Theta}\|_{[\lambda, \mu]}+\int_{\mu}^{1}(1-s)^{\alpha-2} P_{3}(s) d s+\epsilon_{0} \int_{\mu}^{1}(1-s)^{\alpha-2} d s\right] \\
= & \frac{2+\alpha+T_{0}}{\Gamma(\alpha)} l\|x\|_{*}\left[\left\|\hat{P}_{1}\right\|_{[0, \lambda]}+\frac{\epsilon_{0}}{\alpha-1}\left(1-(1-\lambda)^{\alpha-1}\right)\right. \\
& \left.+\left(P_{2}+\epsilon_{0}\right)\|\hat{\Theta}\|_{[\lambda, \mu]}+\left\|\hat{P}_{3}\right\|_{[\mu, 1]}+\frac{\epsilon_{0}}{\alpha-1}(1-\mu)^{\alpha-1}\right] \\
\leq & \theta_{0} l\left[\left\|\hat{P}_{1}\right\|_{[0, \lambda]}+\frac{\epsilon_{0}}{\alpha-1}\left(1-(1-\lambda)^{\alpha-1}\right)\right. \\
& \left.+\left(P_{2}+\epsilon_{0}\right)\|\hat{\Theta}\|_{[\lambda, \mu]}+\left\|\hat{P}_{3}\right\|_{[\mu, 1]}+\frac{\epsilon_{0}}{\alpha-1}(1-\mu)^{\alpha-1}\right]\|x\|_{*} \\
\leq & \|x\|_{*}
\end{aligned}
$$

and so $\|F x\| \leq\|x\|_{*}<r_{0}$. Also, we can conclude that $\left\|F^{\prime} x\right\| \leq\|x\|_{*}<r_{0}$. Hence, $\|F x\|<r_{0}$ and so $F_{x} \in C$. For the same reason, $F_{y} \in C$. Similar to (7), we conclude that

$$
\begin{aligned}
\left\|F_{x}-F_{y}\right\|_{*} \leq & \max \left\{\frac{3 \alpha}{\Gamma(\alpha)}, \frac{2+\alpha+T_{0}}{\Gamma(\alpha)}\right\}\left[\sum_{i=1}^{5}\left\|\hat{a}_{i}\right\|_{[0, \lambda]}\left(l_{\mu_{i}}+\epsilon_{1}\right)\|x-y\|_{*}^{\mu_{i}}\right. \\
& \left.\left.+\sum_{j=1}^{k_{0}}\left\|\hat{b}_{j}\right\|_{[\lambda, \mu]}\left(q_{j}+\epsilon_{1}\right)\|x-y\|_{*}^{m}+\sum_{i=1}^{4}\left\|\hat{c}_{i}\right\|_{[\mu, 1]}\right]\left(l_{\gamma_{i}}+\epsilon_{1}\right)\|x-y\|_{*}^{\left.\gamma_{i}\right]}\right] \\
\leq & \max \left\{\frac{3 \alpha}{\Gamma(\alpha)}, \frac{2+\alpha+T_{0}}{\Gamma(\alpha)}\right\}\left[\sum_{i=1}^{5}\left\|\hat{a}_{i}\right\|_{[0, \lambda]}\left(l_{\mu_{i}}+\epsilon_{1}\right)\|x-y\|_{*}\right. \\
& \left.\left.+\sum_{j=1}^{k_{0}}\left\|\hat{b}_{j}\right\|_{[\lambda, \mu]}\left(q_{j}+\epsilon_{1}\right)\|x-y\|_{*}+\sum_{i=1}^{4}\left\|\hat{c}_{i}\right\|_{[\mu, 1]}\right]\left(l_{\gamma_{i}}+\epsilon_{1}\right)\|x-y\|_{*}\right] \\
= & \max \left\{\frac{3 \alpha}{\Gamma(\alpha)}, \frac{2+\alpha+T_{0}}{\Gamma(\alpha)}\right\}\left[\sum_{i=1}^{5}\left\|\hat{a}_{i}\right\|_{[0, \lambda]}\left(l_{\mu_{i}}+\epsilon_{1}\right)\right. \\
& \left.\left.+\sum_{j=1}^{k_{0}}\left\|\hat{b}_{j}\right\|_{[\lambda, \mu]}\left(q_{j}+\epsilon_{1}\right)+\sum_{i=1}^{4}\left\|\hat{c}_{i}\right\|_{[\mu, 1]}\right]\left(l_{\gamma_{i}}+\epsilon_{1}\right)\right]\|x-y\|_{*}
\end{aligned}
$$

whenever $\|x-y\|_{*}<\epsilon_{1}$. Thus, $\|x-y\|_{*} \leq\|x\|_{*}+\|y\|_{*} \leq \epsilon_{0}$ whenever $x, y \in C$. Hence,

$$
\begin{aligned}
\left\|F_{x}-F_{y}\right\|_{*} \leq & \max \left\{\frac{3 \alpha}{\Gamma(\alpha)}, \frac{2+\alpha+T_{0}}{\Gamma(\alpha)}\right\}\left[\sum_{i=1}^{5}\left\|\hat{a}_{i}\right\|_{[0, \lambda]}\left(l_{\mu_{i}}+\epsilon_{1}\right)\right. \\
& \left.\left.+\sum_{j=1}^{k_{0}}\left\|\hat{b}_{j}\right\|_{[\lambda, \mu]}\left(q_{j}+\epsilon_{1}\right)+\sum_{i=1}^{4}\left\|\hat{c}_{i}\right\|_{[\mu, 1]}\right]\left(l_{\gamma_{i}}+\epsilon_{1}\right)\right]\|x-y\|_{*} \\
= & \psi\left(\|x-y\|_{*}\right),
\end{aligned}
$$


where

$$
\begin{aligned}
\psi(t)= & \max \left\{\frac{3 \alpha}{\Gamma(\alpha)}, \frac{2+\alpha+T_{0}}{\Gamma(\alpha)}\right\}\left[\sum_{i=1}^{5}\left\|\hat{a}_{i}\right\|_{[0, \lambda]}\left(l_{\mu_{i}}+\epsilon_{1}\right)\right. \\
& +\sum_{j=1}^{k_{0}}\left\|\hat{b}_{j}\right\|_{[\lambda, \mu]}\left(q_{j}+\epsilon_{1}\right) \\
& \left.\left.+\sum_{i=1}^{4}\left\|\hat{c}_{i}\right\|_{[\mu, 1]}\right]\left(l_{\gamma_{i}}+\epsilon_{1}\right)\right] t .
\end{aligned}
$$

Note that $\psi \in \Psi$. Now by using Theorem $6, F$ has a fixed point and so the pointwise defined problem (1) has a solution.

Example 2 Consider the problem

$$
D^{\frac{7}{2}} x(t)+f\left(t, x(t), x^{\prime}(t), D^{\frac{1}{2}} x(t), \int_{0}^{t} x(\xi) d \xi\right)=0
$$

with boundary conditions $x(0)=x^{\prime}\left(\frac{1}{3}\right), x(1)=x^{\prime}\left(\frac{1}{2}\right)$ and $x^{\prime \prime}(0)=0$, where

$$
f\left(t, x_{1}, \ldots, x_{4}\right)= \begin{cases}f_{1}\left(t, x_{1}, \ldots, x_{4}\right):=t^{2}\left(\sum_{i=1}^{4} x_{i}(s)\right), & t \in[0,0.7), \\ f_{2}\left(t, x_{1}, \ldots, x_{4}\right):=\frac{0.1}{p(t)} \sum_{i=1}^{4} \frac{\left|x_{i}(t)\right|}{1+\left|x_{i}(t)\right|}, & \frac{t}{3} \in[0.7,0.7] \\ f_{3}\left(t, x_{1}, \ldots, x_{4}\right):=t\left(\sum_{i=1}^{4} x_{i}(s)\right), & t \in[0.9,1]\end{cases}
$$

and

$$
p(t)= \begin{cases}0, & t \in[0.2,0.9] \cap Q \\ t, & t \in[0.2,0.9] \cap Q^{c}\end{cases}
$$

Put $a_{i}(t)=a(t)=t^{2}, b_{j}(t)=b(t)=\frac{0.1}{p(t)}$ and $c_{i}(t)=c(t)=t\left(\right.$ for $\left.1 \leq i \leq 4, k_{0}=1\right)$. Then we have $\left|f_{1}\left(t, x_{1}, \ldots, x_{4}\right)-f_{1}\left(t, y_{1}, \ldots, y_{4}\right)\right| \leq t^{2} \sum_{i=1}^{4}\left|x_{i}-y_{i}\right|=a(t) \sum_{i=1}^{4} \phi\left(\left|x_{i}-y_{i}\right|\right)$,

$$
\left|f_{3}\left(t, x_{1}, \ldots, x_{4}\right)-f_{3}\left(t, y_{1}, \ldots, y_{4}\right)\right| \leq t \sum_{i=1}^{4}\left|x_{i}-y_{i}\right|=c(t) \sum_{i=1}^{4} \Phi\left(\left|x_{i}-y_{i}\right|\right)
$$

and

$$
\left|f_{2}\left(t, x_{1}, \ldots, x_{4}\right)-f_{2}\left(t, y_{1}, \ldots, y_{4}\right)\right| \leq t^{2} \sum_{i=1}^{4}\left|x_{i}-y_{i}\right|=b(t) \sum_{i=1}^{4} H(|x-y|, \ldots,|x-y|),
$$

where $\phi(z)=z, \Phi(z)=z$ and $H\left(z_{1}, \ldots, z_{4}\right)=z_{1}+\cdots+z_{4}$. Put $\mu_{i}=\gamma_{i}=m=1$. Then we have $\lim _{z \rightarrow 0^{+}} \frac{\phi(z)}{z}=1, \lim _{z \rightarrow 0^{+}} \frac{\Phi(z)}{z}=1$ and $\lim _{z \rightarrow 0^{+}} \frac{H_{j}(z, z, z, z)}{z}=1$. Also, $\lim _{\max }\left|x_{i}\right| \rightarrow 0 \frac{\left|f_{1}\left(t, x_{1}, \ldots, x_{4}\right)\right|}{\max \left|x_{i}\right|}=$ $4 t^{2}=P_{1}(t), \lim _{\max }\left|x_{i}\right| \rightarrow 0 \frac{\left|f_{3}\left(t, x_{1}, \ldots, x_{4}\right)\right|}{\max \left|x_{i}\right|}=4 t=P_{3}(t)$ and $\left|f_{2}\left(t, x_{1}, \ldots, x_{4}\right)\right| \leq \Theta(t) \Lambda\left(x_{1}, \ldots, x_{4}\right)$, where $\Theta(t)=p(t)$ and $\Lambda\left(x_{1}, \ldots, x_{4}\right)=\sum_{i=1}^{5} \frac{\left|x_{i}\right|}{1+\left|x_{i}\right|}$. It is easy to see that $\phi, \Phi, H$ and $\Lambda$ satisfy 
the conditions of Theorem 6 and $\lim _{x \rightarrow 0^{+}} \frac{\Lambda(x, x, x, x)}{x}=4:=P_{2}$. Also, we have

$$
\begin{aligned}
& \max \left\{\frac{3 \alpha}{\Gamma(\alpha)}, \frac{2+\alpha+T_{0}}{\Gamma(\alpha)}\right\} \cdot \max \left\{\sum_{i=1}^{5}\left\|\hat{a}_{i}\right\|_{[0, \lambda]}\left(l_{\mu_{i}}\right)+\sum_{j=1}^{k_{0}}\left\|\hat{b}_{j}\right\|_{[\lambda, \mu]} q_{j}\right. \\
& \left.\left.\quad+\sum_{i=1}^{4}\left\|\hat{c}_{i}\right\|_{[\mu, 1]}\right]\left(l_{\gamma_{i}}\right), \max \left\{1, \frac{1}{\Gamma(2-\beta)}, m_{0}\right\}\left[\left\|\hat{P}_{1}\right\|_{[0, \lambda]}+P_{2}\|\Theta\|_{[\lambda, \mu]}+\left\|\hat{P}_{3}\right\|_{[\mu, 1]}\right]\right\} \\
& \leq \frac{\frac{21}{2}}{\frac{15 \sqrt{\pi}}{8}} \max \{0.19+0.005+0.9,1.13[0.19+0.02+0.9]\}<1 .
\end{aligned}
$$

By using Theorem 6, the pointwise defined problem has a solution.

\section{Conclusion}

It is very important that we increase our abilities of natural phenomenon modeling. In this way, it is better we investigate different types of high order fractional integro-differential equations or new type model ones. One of the new models is described by the three step crisis fractional integro-differential equations which have been introduced recently. In this work, we reviewed the existence of solutions for a three step crisis fractional integrodifferential equation under some boundary conditions.

\section{Acknowledgements}

The second and third authors were supported by Azarbaijan Shahid Madani University. The authors express their gratitude to the unknown referees for their helpful suggestions, which improved the final version of this paper.

\section{Funding}

Not applicable.

\section{Availability of data and materials}

Data sharing not applicable to this paper as no datasets were generated or analyzed during the current study.

\section{Competing interests}

The authors declare that they have no competing interests.

\section{Authors' contributions}

Each of the authors contributed to each part of this study equally and approved of the final version of the manuscript.

\section{Author details}

${ }^{1}$ Department of Mathematics, Cankaya University, Ankara, Turkey. ${ }^{2}$ Institute of Space Sciences, Magurele-Bucharest,

Romania. ${ }^{3}$ Department of Mathematics, Azarbaijan Shahid Madani University, Tabriz, Iran.

\section{Publisher's Note}

Springer Nature remains neutral with regard to jurisdictional claims in published maps and institutional affiliations.

Received: 5 January 2019 Accepted: 9 April 2019 Published online: 24 April 2019

\section{References}

1. Abdulnaby, Z.E., Ibrahim, R.W., Kilicman, A.: Some properties for integro-differential operator defined by a fractional formal. SpringerPlus 5, 893 (2016)

2. Agarwal, R.P., Baleanu, D., Hedayati, V., Rezapour, Sh.: Two fractional derivative inclusion problems via integral boundary condition. Appl. Math. Comput. 257, 205-212 (2015)

3. Agarwal, R.P., O'Regan, D., Stanek, S.: Positive solutions for Dirichlet problems of singular nonlinear fractional differential equations. J. Math. Anal. Appl. 371, 57-68 (2010)

4. Agarwal, R.P., O'Regan, D., Stanek, S.: Positive solutions for mixed problems of singular fractional differential equations. Math. Nachr. 285, 27-41 (2012)

5. Alsaedi, A., Baleanu, D., Etemad, S., Rezapour, Sh.: On coupled systems of time-fractional differential problems by using a new fractional derivative. J. Funct. Spaces 2016, Article ID 4626940 (2016)

6. Aydogan, S.M., Baleanu, D., Mousalou, A., Rezapour, Sh.: On high order fractional integro-differential equations including the Caputo-Fabrizio derivative. Bound. Value Probl. 2018, 90 (2018) 
7. Aydogan, S.M., Baleanu, D., Mousalou, A., Rezapour, Sh.: On approximate solutions for two higher-order Caputo-Fabrizio fractional integro-differential equations. Adv. Differ. Equ. 2017, 251 (2017)

8. Bai, Z., Qui, T.: Existence of positive solution for singular fractional differential equation. Appl. Math. Comput. 215, 2761-2767 (2009)

9. Bai, Z., Sun, W., Zhang, W.: Positive solutions for boundary value problems of singular fractional differential equations. Abstr. Appl. Anal. 2013, Article ID 129640 (2013)

10. Baleanu, D., Agarwal, R.P., Mohammadi, H., Rezapour, Sh.: Some existence results for a nonlinear fractional differential equation on partially ordered Banach spaces. Bound. Value Probl. 2013, 112 (2013)

11. Baleanu, D., Ghafarnezhad, Kh., Rezapour, Sh., Shabibi, M.: On the existence of solutions of a three steps crisis integro-differential equation. Adv. Differ. Equ. 2018, 135 (2018)

12. Baleanu, D., Mohammadi, H., Rezapour, Sh.: On a nonlinear fractional differential equation on partially ordered metric spaces. Adv. Differ. Equ. 2013, 83 (2013)

13. Baleanu, D., Mohammadi, H., Rezapour, Sh.: Some existence results on nonlinear fractional differential equations. Philos. Trans. R. Soc. A 371, 20120144 (2013)

14. Baleanu, D., Mohammadi, H., Rezapour, Sh.: The existence of solutions for a nonlinear mixed problem of singular fractional differential equations. Adv. Differ. Equ. 2013, 359 (2013)

15. Baleanu, D., Mousalou, A., Rezapour, Sh.: A new method for investigating approximate solutions of some fractional integro-differential equations involving the Caputo-Fabrizio derivative. Adv. Differ. Equ. 2017, 51 (2017)

16. Baleanu, D., Mousalou, A., Rezapour, Sh.: The extended fractional Caputo-Fabrizio derivative of order $0 \leq \sigma<1$ on $C_{\mathbb{R}}[0,1]$ and the existence of solutions for two higher-order series-type differential equations. Adv. Differ. Equ. 2018 255 (2018)

17. Baleanu, D., Mousalou, A., Rezapour, Sh.: On the existence of solutions for some infinite coefficient-symmetric Caputo-Fabrizio fractional integro-differential equations. Bound. Value Probl. 2017, 145 (2017)

18. Ibrahim, R.W.: The fractional differential polynomial neural network for approximation of functions. Entropy 15(10), 4188-4198 (2013)

19. Ibrahim, R.W., Jalab, H.A.: Existence of the solution of fractional integral inclusion with time delay. Miskolc Math. Notes 11(2), 139-150 (2010)

20. Ibrahim, R.W., Kilicman, A., Damag, F.H.: Existence and uniqueness for a class of iterative fractional differential equations. Adv. Differ. Equ. 2015, 78 (2015)

21. Momani, Sh., Ibrahim, R.W.: On a fractional integral equation of periodic functions involving Weyl-Riesz operator in Banach algebras. J. Math. Anal. Appl. 339, 1210-1219 (2008)

22. Samet, B., Vetro, C., Vetro, P.: Fixed point theorems for $\alpha$ - $\psi$-contractive type mappings. Nonlinear Anal. 72, 2154-2165 (2012)

23. Samko, S.G., Kilbas, A.A., Marichev, O.I.: Fractional Integral and Derivative: Theory and Applications. Gordon \& Breach, New York (1993)

24. Schauder, J.: Der fixpunktsatz in funktionalraumen. Stud. Math. 2, 171-180 (1930)

\section{Submit your manuscript to a SpringerOpen ${ }^{\circ}$ journal and benefit from:}

- Convenient online submission

- Rigorous peer review

- Open access: articles freely available online

- High visibility within the field

- Retaining the copyright to your article

Submit your next manuscript at $\boldsymbol{~ s p r i n g e r o p e n . c o m ~}$ 